\title{
THE VARIANCE-OPTIMAL MARTINGALE MEASURE FOR CONTINUOUS PROCESSES
}

\author{
F. Delbaen and W. Schachermayer * \\ Department of Mathematics, Vrije Universiteit Brussel \\ Institut für Statistik, Universität Wien \\ April 95 (first version April 94)
}

\begin{abstract}
We prove that for continuous stochastic processes $S$ based on $(\Omega, \mathcal{F}, \mathbb{P})$ for which there is an equivalent martingale measure $\mathbb{Q}^{0}$ with square-integrable density $d \mathbb{Q}^{\circ} / d \mathbb{P}$ we have that the so-called "variance optimal" martingale measure $\mathbb{Q}^{o p t}$ for which the density $d \mathbb{Q}^{o p t} / d \mathbb{P}$ has minimal $L^{2}(\mathbb{P})$-norm is automatically equivalent to $\mathbb{P}$.
\end{abstract}

The result is then applied to an approximation problem arising in Mathematical Finance.

\section{INTRODUCTION}

Let $S=\left(S_{t}\right)_{t \in \mathbb{R}_{+}}$be an $\mathbb{R}^{d}$-valued semi-martingale based on $\left(\Omega, \mathcal{F},\left(\mathcal{F}_{t}\right)_{t \in \mathbb{R}_{+}}, \mathbb{P}\right)$ which in most of this paper will be assumed to be continuous. The process $S$ may be interpreted to model the discounted price of $d$ stocks.

A very important tool in Mathematical Finance is to replace the original measure $\mathbb{P}$ by an equivalent local martingale measure $\mathbb{Q}$, sometimes also called a risk-neutral measure. More formally we denote as in [DS $94 \mathrm{a}]$ by

$$
\mathcal{M}(\mathbb{P})=\{\mathbb{Q} \ll \mathbb{P}: \mathbb{Q} \text { is a probability measure and } S \text { is a } \mathbb{Q} \text {-local martingale }\}
$$

the set of all probability measures $\mathbb{Q}$ on $\mathcal{F}$ which are absolutely continuous with respect to $\mathbb{P}$ and such that $S$ becomes a local martingale under $\mathbb{Q}$. By

$$
\mathcal{M}^{e}(\mathbb{P})=\{\mathbb{Q} \sim \mathbb{P}: \mathbb{Q} \text { is a probability measure and } S \text { is a } \mathbb{Q} \text {-local martingale }\}
$$

we denote the subset of $\mathcal{M}(\mathbb{P})$ formed by the probability measures $\mathbb{Q} \in \mathcal{M}(\mathbb{P})$ which are equivalent to $\mathbb{P}$.

A basic problem in Mathematical Finance is to determine (i.e., find necessary and sufficient conditions on $S$ ) whether or not $\mathcal{M}^{e}(\mathbb{P})$ is non-empty. This issue is settled by the Fundamental Theorem of Asset Pricing, where some kind of no arbitrage assumption is needed to insure that $\mathcal{M}^{e}(\mathbb{P}) \neq \emptyset$. We refer to [DS 94] for a general version of this theorem and for a detailed account on related work on this problem, starting from the seminal papers [HK 79], [HP 81], [Kre 81].

Once it is established that the set $\mathcal{M}^{e}(\mathbb{P})$ of equivalent local martingale measures is non-empty the question arises, which element $\mathbb{Q}$ in $\mathcal{M}^{e}(\mathbb{P})$ is the "most natural" choice and how the choice of $\mathbb{Q}$ is related to the pricing and hedging of a given contingent claim, i.e., an $\mathcal{F}$-measurable random variable $f$. The term "most natural", of course, depends on the context. Note that in the general setting of the

1980 Mathematics Subject Classification (1985 Revision). 90A09,60G44,46N10,47N10,60H05,60G40.

Key words and phrases. Equivalent Martingale Measure, Pricing by Arbitrage, Optimal Measure, Risk Neutral Measure, Representing Measure, Mathematical Finance.

Part of this research was supported by the European Community Stimulation Plan for Economic Science contract Number SPES-CT91-0089. . 
Fundamental Theorem of Asset Pricing (as presented in [DS 94]) it does not make sense to ask for a "most natural" element of $\mathcal{M}^{e}(\mathbb{P})$ as this setting is invariant under changes of equivalent measures. Hence the question is as meaningful (or meaningless) as asking "what is the most natural point in a convex set".

But once we fix the original measure $\mathbb{P}$, one may ask which element $\mathbb{Q} \in \mathcal{M}^{e}(\mathbb{P})$ is most natural (relative to this measure $\mathbb{P}$ ). In the applications in Mathematical Finance and in particular in Actuarial Mathematics one often has quite a good knowledge of what the measure $\mathbb{P}$, which describes the "real" world, should be. For example, insurance companies usually have a very precise knowledge of the "true" mortality in their (life insurance) portfolios, which is modeled by $\mathbb{P}$ ("mortality tables of second order"), while for calculating premia and reserves they use substantially different probability measures $\mathbb{Q}$ ("mortality tables of first order").

If we have a good reason to fix the measure $\mathbb{P}$, it makes sense to ask for the element $\mathbb{Q} \in \mathcal{M}^{e}(\mathbb{P})$ which is "closest" to $\mathbb{P}$. So far there have been mainly two notions of "closest" element considered. For continuous semi-martingales H. Föllmer and M. Schweizer [FS 91] called the element $\mathbb{Q} \in \mathcal{M}^{e}(\mathbb{P})$ which minimizes the relative entropy

$$
H(\mathbb{Q} \mid \mathbb{P})=\int \log \left(\frac{d \mathbb{Q}}{d \mathbb{P}}\right) d \mathbb{Q}
$$

the minimal martingale measure. If $S$ is continuous and its Doob-Meyer decomposition is of the form $S=M+A=M+\alpha^{\prime} \cdot\langle M\rangle$ (where ' denotes transposition in $\mathbb{R}^{d}$ ) for some predictable process $\alpha$, then the density of the minimal martingale measure $\mathbb{Q}^{\text {min }}$ is given by the Girsanov-type formula

$$
\begin{aligned}
\frac{d \mathbb{Q}^{\text {min }}}{d \mathbb{P}} & =\mathcal{E}\left(-\alpha^{\prime} \cdot M\right)_{\infty} \\
& =\exp \left(-\int_{0}^{\infty} \alpha_{t}^{\prime} d M_{t}-\frac{1}{2} \int_{0}^{\infty} d\left\langle\alpha^{\prime} \cdot M\right\rangle_{t}\right),
\end{aligned}
$$

provided this measure exists, i.e. provided that $\mathcal{E}(-\alpha \cdot M)_{t}$ is a strictly positive uniformly integrable martingale (compare [F-S 91]). This formula is particularly appealing if we know from arguments involving martingale representation (e.g., in the case of a "Brownian" setting) that there is at most one equivalent (local) martingale measure. In this case one simply has to verify whether the process $\mathcal{E}(-\alpha \cdot M)_{t}$ is a uniformly integrable strictly positive martingale or not; several sufficient conditions, e.g. Novikov's and Kazamaki's condition [KS 91] [RY 91], are known to guarantee this.

However, it turns out that the Girsanov-type formula above may go astray, although there may be equivalent martingale measures around: in [Sch 93] and [DS 95] the authors constructed a continuous process $S=M+\alpha \cdot\langle M\rangle$ such that there exist equivalent martingale measures $\mathbb{Q}$ (even with $\frac{d \mathbb{Q}}{d \mathbb{P}}$ uniformly bounded) but nevertheless the local martingale $\mathcal{E}(-\alpha \cdot M)$ is not uniformly integrable. Hence, despite of many appealing properties (see, e.g. [FS 91], [DR 91], [AS 93], [Schw 92a], [Schä 94]) one cannot rely on the existence of the minimal martingale measure, even if $S$ is continuous and models a perfectly arbitrage-free market.

Another natural approach is to look at the element of $\mathcal{M}^{e}(\mathbb{P})$ of smallest $L^{2}$-norm, in other words to look for the element $\mathbb{Q} \in \mathcal{M}^{e}(\mathbb{P})$ which minimizes

$$
D(\mathbb{Q}, \mathbb{P})=\left\|\frac{d \mathbb{Q}}{d \mathbb{P}}\right\|_{L^{2}(\mathbb{P})}=\left(\text { Variance }\left(\frac{d \mathbb{Q}}{d \mathbb{P}}\right)+1\right)^{\frac{1}{2}}
$$

provided such an element exists (uniqueness will follow from strict convexity of the norm of $L^{2}$ ). We refer to [Schw 92a] for the name "variance-optimal" and for the relevance and history of this idea.

To introduce this concept in a precise way it is convenient to introduce the notion of "signed local martingale measures" which was introduced by S. Müller [M 85] (compare [Schw 92a], [AS 92]). Let $K_{0}$ denote the subspace of $L^{\infty}(\Omega, \mathcal{F}, \mathbb{P})$ spanned by the "simple" stochastic integrals of the form

$$
f=h^{\prime}\left(S_{T_{2}}-S_{T_{1}}\right)
$$


where $T_{1} \leq T_{2}$ are stopping times such that the stopped process $S^{T_{2}}$ is bounded and $h$ is a bounded $\mathbb{R}^{d}$-valued $\mathcal{F}_{T_{1}}$-measurable function. Obviously, if $S$ is assumed to be a locally bounded cadlag semimartingale, a probability measure $\mathbb{Q}$ on $\mathcal{F}$ is a local martingale measure for $S$ iff $\mathbb{Q}$ vanishes on $K_{0}$, i.e.,

$$
\mathbf{E}_{\mathbb{Q}}[f]=\mathbf{E}\left[\frac{d \mathbb{Q}}{d \mathbb{P}} f\right]=0 \quad \forall f \in K_{0}
$$

Identifying absolutely continuous measures with their Radon-Nikodym derivatives — which we shall freely do throughout this paper without further notice - this leads to the subsequent concept.

1.1 Definition. The set of signed local martingale measures for the process $S$ is the affine subspace $\mathcal{M}^{s}(\mathbb{P})$ of $L^{1}(\mathbb{P})$

$$
\mathcal{M}^{s}(\mathbb{P})=\left\{g \in L^{1}(\mathbb{P}): \mathbf{E}[g f]=0 \text { for } f \in K_{0}, \text { and } \mathbf{E}[g]=1\right\}
$$

i.e., $\mathcal{M}^{s}(\mathbb{P})$ is the intersection of the annihilator of $K_{0}$ with the set $H=\{g: \mathbb{E}[g]=1\}$. Note that $H$ is an affine hyperplane (i.e., an affine subspace of codimension 1 ) of $L^{1}(\mathbb{P})$ and that $H$ is spanned by (the densities of ) the probability measures in $L^{1}(\mathbb{P})$, i.e., $H$ is the smallest affine subspace of $L^{1}(\mathbb{P})$ containing these probability measures.

Obviously $\mathcal{M}(\mathbb{P})$ (resp. $\mathcal{M}^{e}(\mathbb{P})$ ) is the intersection of $\mathcal{M}^{s}(\mathbb{P})$ with the positive (resp. strictly positive) orthant of $L^{1}(\mathbb{P})$. Noting that the intersection of $\mathcal{M}^{s}(\mathbb{P})$ with $L^{2}(\mathbb{P})$ is closed in the norm of $L^{2}(\mathbb{P})$ and that a (non-empty) closed, convex subset of $L^{2}(\mathbb{P})$ has a unique element of minimal norm, we can now define the central concept of this paper:

1.2 Definition. [Schw $92 \mathrm{a}]$ If $\mathcal{M}^{s}(\mathbb{P}) \cap L^{2}(\mathbb{P}) \neq \emptyset$ we call the element of $\mathcal{M}^{s}(\mathbb{P})$ with minimal $L^{2}(\mathbb{P})$-norm the variance-optimal signed local martingale measure for the process $S$.

Why do we have to pass to the space of signed local martingale measures? As observed in [AS 92] one may easily construct examples (the underlying probability space $\Omega$ may be chosen to consist of 3 elements only) such that the variance-optimal - as well as the minimal - martingale measure is only a signed measure, i.e., assumes negative values. This phenomenon is due to the fact that if $S$ has jumps the stochastic exponential $\mathcal{E}(-\alpha \cdot M)$ may become negative.

On the other hand, for continuous processes the stochastic exponential $\mathcal{E}(-\alpha \cdot M)$ is certainly non-negative, hence the minimal local martingale measure - if it exists - certainly is a probability measure.

This triggered the question, whether for continuous processes we always have that the variance-optimal local martingale measure (whose existence follows from the very weak assumption $\mathcal{M}^{s}(\mathbb{P}) \cap L^{2}(\mathbb{P}) \neq \emptyset$, compare Lemma 2.1 below) is automatically non-negative. In fact, it turns out that it is automatically strictly positive, i.e., equivalent to $\mathbb{P}$, provided that the obviously necessary requirement $\mathcal{M}^{e}(\mathbb{P}) \cap L^{2}(\mathbb{P}) \neq$ $\emptyset$ is satisfied.

1.3 Main Theorem. Let $S$ be a continuous, $\mathbb{R}^{d}$-valued semi-martingale and suppose that $\mathcal{M}^{e}(\mathbb{P}) \cap$ $L^{2}(\mathbb{P}) \neq \emptyset$, i.e., there is at least one equivalent local martingale measure with square-integrable density. Then the variance-optimal measure $\mathbb{Q}^{\text {opt }}$ is a probability measure equivalent to $\mathbb{P}$.

We finish this introduction by pointing out that M. Schweizer [Schw 94] showed independently that in the setting of the main theorem we have that $\mathbb{Q}^{\text {opt }}$ is a $\mathbb{P}$-absolutely continuous probability measure, i.e., $\mathbb{Q}^{\text {opt }} \in \mathcal{M}(\mathbb{P})$ (as opposed to the stronger conclusion $\mathbb{Q}^{\text {opt }} \in \mathcal{M}^{e}(\mathbb{P})$ in the preceding theorem; compare Theorem 3.1 below). 


\section{Notation and PRELIminary RESUlts}

By $S=\left(S_{t}\right)_{t \in \mathbb{R}_{+}}$we denote an $\mathbb{R}^{d}$-valued cadlag locally bounded semi-martingale. We choose $\mathbb{R}_{+}$as the time index set as this setting covers the most general case. The process $S$ will be based on a filtered probability space $\left(\Omega, \mathcal{F},\left(\mathcal{F}_{t}\right)_{t \in \mathbb{R}_{+}}, \mathbb{P}\right)$ satisfying the usual conditions. By $\widehat{K}_{0}$ we denote the closure of $K_{0}$ in $L^{2}(\Omega, \mathcal{F}, \mathbb{P})$ and by $\widehat{K}$ the closure of the span of $K_{0}$ and the constants in $L^{2}(\mathbb{P})$ :

$$
\widehat{K}=\overline{\operatorname{span}\left(K_{0}, 1\right)} \text {. }
$$

The following easy lemma shows the orthogonality relation between the space $K_{0}$ of simple stochastic integrals on $S$ and the affine space of signed local martingale measures for $S$.

\subsection{Lemma.}

(a) $\mathcal{M}^{s}(\mathbb{P}) \cap L^{2}(\mathbb{P})$ is non-empty iff $\widehat{K}_{0}$ does not contain the constant function 1 .

(b) $A$ (signed) measure $\mathbb{Q}$ on $\mathcal{F}$ with $\frac{d \mathbb{Q}}{d \mathbb{P}} \in L^{2}(\mathbb{P})$ is in $\mathcal{M}^{s}(\mathbb{P})$ iff $\mathbb{E}_{\mathbb{Q}}[\cdot]$ vanishes on $\widehat{K}_{0}$ and equals 1 on the constant function 1.

(c) If $\mathcal{M}^{s}(\mathbb{P}) \cap L^{2}(\mathbb{P}) \neq \emptyset$, then $\mathbb{Q}^{\text {opt }}$ is the unique element of $\widehat{K}$ vanishing on $\widehat{K}_{0}$ and equaling 1 on the constant function 1. (Here we identify the measure $\mathbb{Q}^{\text {cot }}$ with the linear functional $\mathbb{E}_{\mathbb{Q}}[\cdot]$ and linear functionals on $L^{2}(\mathbb{P})$ with elements of $L^{2}(\mathbb{P})$.)

Proof. The assertion (b) is an immediate consequence of the very definition of the space $\mathcal{M}^{s}(\mathbb{P})$ of signed local martingale measures and (a) follows from the fact that the linear functional $\varphi$ on $\hat{K}$ which satisfies $\left.\varphi\right|_{\widehat{K}_{0}}=0$ and $\varphi(1)=1$ is well defined and continuous on the closed subspace $\widehat{K}$ of $L^{2}(\mathbb{P})$ iff $1 \notin \widehat{K}_{0}$. Finally (c) is implied by the elementary fact that the extension of $\varphi$ from $\widehat{K}$ to $L^{2}(\mathbb{P})$ with minimal norm vanishes on the orthogonal complement of $\widehat{K}$.

q.e.d.

In the sequel we shall assume that $\mathcal{M}^{s}(\mathbb{P}) \cap L^{2}(\mathbb{P}) \neq \emptyset$ so that the (signed) variance-optimal local martingale measure, denoted by $\mathbb{Q}^{o p t}$, exists. We denote by $Z_{\infty}^{o p t}$ the Radon-Nikodym derivative $\frac{d \mathbb{Q}^{o p t}}{d \mathbb{P}}$ and by $Z_{t}^{\text {opt }}$ the Radon-Nikodym derivative of the restrictions to $\mathcal{F}_{t}$ so that $\left(Z_{t}^{\text {opt }}\right)_{t \in \mathbb{R}_{+}}$is a $\mathbb{P}$-martingale converging to $Z_{\infty}^{\text {opt }}$ in $L^{2}(\mathbb{P})$.

In most of the paper we shall assume that $\mathcal{M}^{e}(\mathbb{P}) \cap L^{2}(\mathbb{P}) \neq \emptyset$ and fix some element $\mathbb{Q}^{0} \in \mathcal{M}^{e}(\mathbb{P}) \cap L^{2}(\mathbb{P})$, i.e., an arbitrarily chosen equivalent local martingale measure with square-integrable density $Z_{\infty}^{0}=\frac{d \mathbb{Q}^{0}}{d \mathbb{P}}$. Again we denote by $Z_{t}^{0}$ the conditional expectation of $Z_{\infty}^{0}$ with respect to $\mathcal{F}_{t}$.

We also associate to $Z_{\infty}^{\text {opt }}$ the $\mathbb{Q}^{0}$-martingale

$$
\hat{Z}_{t}^{o p t}=\mathbf{E}_{\mathbb{Q}^{0}}\left[Z_{\infty}^{o p t} \mid \mathcal{F}_{t}\right]
$$

The next lemma shows that the process $\hat{Z}^{\circ p t}$ is independent of the choice of $\mathbb{Q}^{0}$ and may be written as a constant $c$, given by $\left\|Z_{\infty}^{\text {opt }}\right\|_{L^{2}(\mathbb{P})}^{2}$, and a stochastic integral on $S$. This basic fact was already observed in [DR 91], [Schä 94], [Schw 94] in various degrees of generality. We refer to [Schw 94] for an account on these results.

2.2 Lemma. Let $S$ be a locally bounded semi-martingale such that $\mathcal{M}^{e}(\mathbb{P}) \cap L^{2}(\mathbb{P}) \neq \emptyset$ and fix $\mathbb{Q}^{0} \in$ $\mathcal{M}^{e}(\mathbb{P}) \cap L^{2}(\mathbb{P})$.

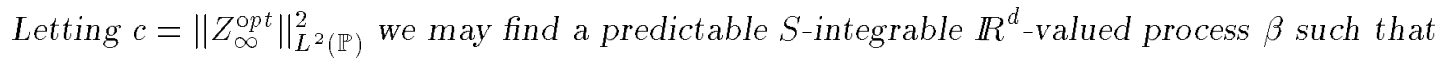

$$
\hat{Z}_{t}^{o p t}=c+\left(\beta^{\prime} \cdot S\right)_{t}
$$


where the stochastic integral $\beta^{\prime} \cdot S$ is well defined and is a uniformly integrable martingale with respect to $\mathbb{Q}^{0}$ as well as with respect to any other measure $\mathbb{Q}^{1} \in \mathcal{M}^{e}(\mathbb{P}) \cap L^{2}(\mathbb{P})$.

The choice of $\beta$ is independent of the choice of $\mathbb{Q}^{0} \in \mathcal{M}^{e}(\mathbb{P}) \cap L^{2}(\mathbb{P})$.

Proof. Let $f$ be in $K=\operatorname{span}\left(K_{0}, 1\right)$, i.e.,

$$
f=\delta+\sum_{i=1}^{n} h_{i}^{\prime}\left(S_{T_{i}^{2}}-S_{T_{i}^{1}}\right)
$$

where $\delta \in \mathbb{R}$ and where $T_{i}^{2} \geq T_{i}^{1}$ are stopping times such that, for $i=1, \ldots, n$, the process $S^{T_{i}^{2}}$ is bounded and $h_{i}$ is a random variable in $L^{\infty}\left(\Omega, \mathcal{F}_{T_{i}^{1}}, \mathbb{P} ; \mathbb{R}^{d}\right)$. Clearly the uniformly integrable $\mathbb{Q}^{0}$-martingale

$$
f_{t}=\mathbf{E}_{\mathbb{Q}^{0}}\left[f \mid \mathcal{F}_{t}\right]
$$

is a simple stochastic integral on $S$ (plus a constant) as

$$
\begin{aligned}
f_{t} & =\delta+\sum_{i=1}^{n} h_{i}^{\prime}\left(S_{T_{i}^{2} \wedge t}-S_{T_{i}^{1} \wedge t}\right) \\
& =\delta+(H . S)_{t}
\end{aligned}
$$

where $H=\sum_{i=1}^{n} h_{i} \mathbb{\rrbracket} \rrbracket T_{i}^{1}, T_{i}^{2} \rrbracket$.

By Lemma 2.1 (c) there is a sequence $\left(f_{j}\right)_{j=1}^{\infty} \in K$ converging to $Z_{\infty}^{\text {opt }}$ in $L^{2}(\mathbb{P})$, whence by the CauchySchwarz inequality, in $L^{1}\left(\mathbb{Q}^{0}\right)$. If $\delta_{j}$ denotes the real numbers in the representation of $f_{j}$ as stochastic integrals we get

$$
\begin{aligned}
\lim _{j \rightarrow \infty} \delta_{j} & =\lim _{j \rightarrow \infty} \mathbf{E}_{\mathbb{Q}^{0}}\left[f_{j}\right] \\
& =\lim _{j \rightarrow \infty} \mathbf{E}_{\mathbb{P}}\left[Z_{\infty}^{0} f_{j}\right] \\
& =\mathbf{E}_{\mathbb{P}}\left[Z_{\infty}^{0} Z_{\infty}^{\text {opt }}\right] \\
& =\mathbf{E}_{\mathbb{P}}\left[\left(Z_{\infty}^{\text {opt }}\right)^{2}\right]=\left\|Z_{\infty}^{\circ p t}\right\|_{L^{2}(\mathbb{P})}^{2}
\end{aligned}
$$

The last line follows from the fact that, by the optimality of $Z_{\infty}^{\text {opt }}$, the random variable $Z_{\infty}^{0}-Z_{\infty}^{\text {opt }}$ is orthogonal to $Z_{\infty}^{\text {opt }}$ in $L^{2}(\mathbb{P})$.

The random variables $\left(f_{j}-\delta_{j}\right)_{j=1}^{\infty}$ converge in $L^{1}\left(\mathbb{Q}^{0}\right)$ to $Z_{\infty}^{\text {opt }}-\left\|Z_{\infty}^{\circ p t}\right\|_{L^{2}(\mathbb{P})}^{2}$ and we may apply a theorem of Yor ([Yor 78] th. 4.2., for the vector-valued case see [Ja 79], th. 4.60, p. 143) to obtain the desired integrand $\beta$.

As regards the last assertion of the lemma, note that, if we choose instead of $\mathbb{Q}^{0}$ another element $\mathbb{Q}^{1}$ of $\mathcal{M}^{e}(\mathbb{P}) \cap L^{2}(\mathbb{P})$ the process $\beta^{\prime} \cdot S$ remains unchanged and is a $\mathbb{Q}^{1}$ uniformly integrable martingale converging to $Z_{\infty}^{\text {opt }}-\left\|Z_{\infty}^{\text {opt } t}\right\|_{L^{2}(\mathbb{P})}^{2}$ in $L^{1}\left(\mathbb{Q}^{1}\right)$.

q.e.d.

2.3 Corollary. If the semi-martingale $S$ is continuous, the process $\hat{Z}_{t}^{\text {opt }}$ is continuous too.

2.4 Remark. On the other hand, the continuity of $S$ does not imply that the $\mathbb{P}$-martingale $Z_{t}^{\text {opt }}$ is continuous. The following easy example goes back to Harrison-Pliska ([HP 81]; see also [FS 91], ex. 5.13) and may serve as a general source of intuition for the theory developed in section 3 . 
2.5 Example. Let $W=\left(W_{t}\right)_{0 \leq t \leq 2}$ be standard Brownian motion based on $\left(\Omega, \mathcal{F},\left(\mathcal{G}_{t}\right)_{0 \leq t \leq 2}, \mathbb{P}\right)$ and let $r$ be a random variable based on $(\Omega, \mathcal{F}, \mathbb{P})$, independent of $W$, taking the values 0 and 1 with probability $\frac{1}{2}$. For $t<1$ let $\mathcal{F}_{t}=\mathcal{G}_{t}$ and for $t \geq 1$ let $\mathcal{F}_{t}$ be the $\sigma$-algebra generated by $\mathcal{G}_{t}$ and $r$ and define

$$
S_{t}=W_{t}+r(t-1)^{+}
$$

We may and do assume that $\mathcal{G}_{t}$ is the filtration generated by $W$ and that $\mathcal{F}$ is generated by $W$ and $r$.

The process $S$ models the following situation: Before time 1 we simply have Brownian motion; at time 1 a coin is flipped and according to the result the process either continues to be Brownian motion or it becomes Brownian motion with constant drift equal to one. We stop the example at time $t=2$.

In this case we do not have uniqueness of the martingale measures for $S$. Indeed

$$
Z_{\infty}^{(1)}=2 \mathbb{I}\{r=0\}
$$

is the density of a martingale measure $\mathbb{Q}^{1}$ and so is, by Girsanov's formula,

$$
\begin{aligned}
Z_{\infty}^{(2)} & =2 \mathbb{I}\{r=1\} \cdot \exp \left(-\int_{1}^{2} d W_{s}-\frac{1}{2} \int_{1}^{2} d s\right) \\
& =2 \mathbb{I}\{r=1\} \cdot \exp \left(\varphi-\frac{1}{2}\right)
\end{aligned}
$$

where $\varphi$ denotes the standard Gaussian random variable $\varphi=W_{1}-W_{2}$.

The general form of the density $Z$ of a signed martingale measure for $S$ is given by

$$
Z_{\infty}=\lambda Z_{\infty}^{(1)}+(1-\lambda) Z_{\infty}^{(2)}
$$

with $\lambda \in \mathbb{R}$ and $Z_{\infty}$ is the density of a probability measure (resp. an equivalent probability measure) iff $\lambda \in[0,1]$ (resp. $\lambda \in] 0,1[$ ).

Denoting again

$$
Z_{t}=\mathbf{E}\left[Z_{\infty} \mid \mathcal{F}_{t}\right]
$$

the process $Z$ is continuous iff $\lambda=\frac{1}{2}$, in which case $Z$ is the density of the "minimal" martingale measure, as one easily verifies.

As regards the "variance-optimal" martingale measure, note that by elementary calculations we obtain

hence by Pythagoras' theorem

$$
\left\|Z_{\infty}^{(1)}\right\|_{L^{2}(\mathbb{P})}^{2}=2
$$

while

$$
\left\|Z_{\infty}^{(2)}\right\|_{L^{2}(\mathbb{P})}^{2}=2 e,
$$

$$
\left\|\lambda Z_{\infty}^{(1)}+(1-\lambda) Z_{\infty}^{(2)}\right\|_{L^{2}(\mathbb{P})}^{2}=2 \lambda^{2}+2 e(1-\lambda)^{2} .
$$

The value of $\lambda$ which minimizes the above expression is not equal to $\frac{1}{2}$ but equals $\lambda^{\text {opt }}=\frac{e}{e+1}>\frac{1}{2}$ for which we get

$$
\left\|Z_{\infty}^{\mathrm{op} t}\right\|_{L^{2}(\mathbb{P})}^{2}=\left\|\lambda^{\mathrm{opt}} Z_{\infty}^{(1)}+\left(1-\lambda^{\mathrm{opt}}\right) Z_{\infty}^{(2)}\right\|_{L^{2}(\mathbb{P})}^{2}=\frac{2 e}{e+1} .
$$

In particular the (cadlag version of the) $\mathbb{P}$-martingale $Z_{t}^{\text {opt }}$ equals identically 1 for $0 \leq t<1$, while

$$
Z_{1}^{\text {opt }}=\frac{2 e}{e+1} \mathbb{I}\{r=0\}+\frac{2}{e+1} \mathbb{I}\{r=1\},
$$

so that $Z^{\text {opt }}$ has a jump at $t=1$. For $\left.\left.t \in\right] 1,2\right]$ the process $Z_{t}^{\text {opt }}$ is continuous and we also may explicitly calculate it:

$$
Z_{t}^{\mathrm{opt}}=\frac{2 e}{e+1} \mathbb{I}\{r=0\}+\frac{2}{e+1} \mathbb{I}\{r=1\} \exp \left(-\left(W_{t}-W_{1}\right)-\frac{t-1}{2}\right) .
$$




\section{The proof of the Theorem}

Throughout this section we assume that $S$ is a continuous adapted process. We start with the preliminary result that, under very general conditions, $\mathbb{Q}^{\text {opt }}$ is a well-defined probability measure absolutely continuous with respect to $\mathbb{P}$ and with square-integrable density, i.e., $\mathbb{Q}^{\text {opt }} \in \mathcal{M}(\mathbb{P}) \cap L_{+}^{2}(\mathbb{P})$. The more delicate issue of showing that $\mathbb{Q}^{\text {opt }}$ is equivalent to $\mathbb{P}$, i.e., $\mathbb{Q}^{\text {opt }} \in \mathcal{M}^{e}(\mathbb{P}) \cap L_{+}^{2}(\mathbb{P})$, will only be tackled later.

3.1 Theorem. If the adapted stochastic process $S$ is continuous and if the constant Function II is not in $\hat{K}_{0}$ then the variance-optimal measure $\mathbb{Q}^{\text {opt }}$ exists and is in $L^{2}(\mathbb{P})$.

Proof. We cannot make use of the results of lemma 2.2 and hence we cannot state that $Z_{\infty}^{\text {opt }}$ is given by a stochastic integral with respect to the process $S$. We in fact don't even assume that $S$ is a semimartingale. Some approximation is therefore needed. Let $f$ be the orthogonal projection of the constant function 1 onto the space $\widehat{K_{0}}$. From elementary linear algebra it follows that the optimal measure is given by $Z_{\infty}^{\text {opt }}=\frac{1-f}{1-\mathrm{E}[f]}$. Also it is clear that $\mathbf{E}[f]=\mathbf{E}[1 f]=\mathbf{E}\left[f^{2}\right]<1$, proving that $0 \leq \mathbf{E}[f]<1$. Showing that $\mathbb{Q}^{\text {opt }}$ is non-negative, is therefore the same as proving that $f \leq 1$.

Suppose on the contrary the existence of $\varepsilon>0$ such that $\mathbb{P}[f>1+\varepsilon]>\varepsilon$. Take $K$ a simple integrand such that $g=(K \cdot S)_{\infty} \in K_{0}$ and such that $\|g-f\| \leq \eta$ where $\eta \leq \frac{\varepsilon^{3}}{32}$. We may, as easily seen, also suppose that $\left\|1-(K \cdot S)_{\infty}\right\|_{2} \leq 1$, where $\|\cdot\|$ denotes the norm of $L^{2}(\mathbb{P})$. From Cebysev's inequality we deduce that

$$
\begin{aligned}
\mathbb{P}\left[(K \cdot S)_{\infty}>1+\frac{\varepsilon}{2}\right] & \geq \mathbb{P}[f>1+\varepsilon]-\mathbb{P}\left[\left|f-(K \cdot S)_{\infty}\right|>\frac{\varepsilon}{2}\right] \\
& \geq \varepsilon-\frac{4}{\varepsilon^{2}} \eta^{2} \geq \frac{\varepsilon}{2}
\end{aligned}
$$

Define now $T=\inf \left\{t \mid(K \cdot S)_{t}>1\right\}$. Clearly we have that

$$
\begin{aligned}
\left|1-(K \cdot S)_{\infty}\right|^{2} & =\left(1-(K \cdot S)_{T}\right)^{2} \mathbb{I}\{T=\infty\}+\left(1-(K \cdot S)_{\infty}\right)^{2} \mathbb{I}\{T<\infty\} \\
& =\left(1-(K \cdot S)_{T}\right)^{2}+\left(1-(K \cdot S)_{\infty}\right)^{2} \mathbb{I}\{T<\infty\}
\end{aligned}
$$

where the last equality follows from the continuity of $S$. From this we deduce that (denoting by $\|\cdot\|$ the norm of $\left.L^{2}(\mathbb{P})\right)$

$$
\begin{aligned}
\left\|1-(K \cdot S)_{\infty}\right\|^{2} & \geq\left\|1-(K \cdot S)_{T}\right\|^{2}+\int_{T<\infty}\left(1-(K \cdot S)_{\infty}\right)^{2} \\
& \geq\left\|1-(K \cdot S)_{T}\right\|^{2}+\int_{(K \cdot S)_{\infty}>1+\frac{\varepsilon}{2}}\left(1-(K \cdot S)_{\infty}\right)^{2} \\
& \geq\left\|1-(K \cdot S)_{T}\right\|^{2}+\frac{\varepsilon}{2}\left(\frac{\varepsilon}{2}\right)^{2} \\
& \geq\left\|1-(K \cdot S)_{T}\right\|^{2}+\frac{\varepsilon^{3}}{8}
\end{aligned}
$$

On the other hand

$$
\left\|1-(K \cdot S)_{\infty}\right\| \leq\|1-f\|+\eta
$$

and hence, as $\left\|1-(K \cdot S)_{\infty}\right\| \leq 1$,

$$
\begin{aligned}
\|1-f\|^{2} & \geq\left\|1-(K \cdot S)_{\infty}\right\|^{2}-2 \eta \\
& \geq\left\|1-(K \cdot S)_{T}\right\|^{2}+\frac{\varepsilon^{3}}{8}-2 \eta \\
& \geq\left\|1-(K \cdot S)_{T}\right\|^{2}+\frac{\varepsilon^{3}}{16} .
\end{aligned}
$$


These inequalities show that $f$ cannot be the projection of the function 1 .

q.e.d.

Remark. Some of the ideas of the above proof come from [St 90].

From now on we again make the assumption that $\mathcal{M}^{e}(\mathbb{P}) \cap L^{2}(\mathbb{P}) \neq \emptyset$, which implies in particular that $S$ is a semi-martingale. Again we denote by $\mathbb{Q}^{\text {opt }}$ the element of $\mathcal{M}^{s}(\mathbb{P})$ of smallest $L^{2}(\mathbb{P})$-norm, we fix some $\mathbb{Q}^{0} \in \mathcal{M}^{e}(\mathbb{P}) \cap L^{2}(\mathbb{P})$ and we let

$$
\begin{aligned}
Z_{t}^{o p t} & =\mathbf{E}_{\mathbb{P}}\left[\frac{d \mathbb{Q}^{c t}}{d \mathbb{P}} \mid \mathcal{F}_{t}\right] \\
Z_{t}^{0} & =\mathbf{E}_{\mathbb{P}}\left[\frac{d \mathbb{Q}^{0}}{d \mathbb{P}} \mid \mathcal{F}_{t}\right],
\end{aligned}
$$

where, of course, we choose cadlag-versions for the processes $Z^{\circ p}$ and $\mathbb{Z}^{0}$. The density $\frac{d \mathbb{Q}}{d \mathbb{P}}=Z_{\infty}^{\mathrm{opt}}$ is given by $Z_{\infty}^{\circ p t}=\frac{1-f}{1-\mathrm{E}[f]}$ where $f$ is the orthogonal projection of 1 on $\widehat{K_{0}}$. As shown in section 2 the element $f$ is given by a stochastic integral and is of the form $f=(H \cdot S)_{\infty}$ for some predictable process $H$. To show that $\mathbb{Q}^{\text {opt }}$ is equivalent we only need to show that $f<1$ a.s.. Let us put

$$
\begin{aligned}
Y_{t} & =1-(H \cdot S)_{t}=\mathbf{E}_{\mathbb{Q}^{0}}\left[Y_{\infty} \mid \mathcal{F}_{t}\right] \quad \text { where } \quad Y_{\infty}=1-f \\
X_{t} & =\mathbf{E}_{\mathbb{P}}\left[Y_{\infty} \mid \mathcal{F}_{t}\right]=(1-\mathbf{E}[f]) Z_{t}^{\text {opt }} \\
\sigma & =\inf \left\{t \mid Y_{t}=0\right\} \\
T & =\inf \left\{t \mid X_{t}=0\right\}
\end{aligned}
$$

From the previous theorem 3.1 we know already that both processes $Y$ and $X$ are non-negative. We also have that on the stochastic interval $\llbracket \sigma, \infty[$ (resp. $\llbracket T, \infty \llbracket$ ) the process $Y$ (resp. $X$ ) is constant as, by the preceding theorem 3.1, the random variables $X_{\infty}$ and $Y_{\infty}$ are non-negative. Because the process $Y$ is continuous, the stopping time $\sigma$ is clearly predictable; indeed it is announced by the sequence $\sigma_{n}=\inf \left\{t \mid Y_{t} \leq \frac{1}{n+1}\right\} \wedge n$

3.2 Lemma. Let $S$ be a continuous semi-martingale. If the set $\mathcal{M}^{e}(\mathbb{P}) \cap L^{2}(\mathbb{P}) \neq \emptyset$ then $\sigma=T$. Consequently $T$ is predictable.

Proof. (1) On the set $\{\sigma<T\}$ we have

$$
\begin{aligned}
0<X_{\sigma} & =\mathbf{E}\left[X_{\infty} \mid \mathcal{F}_{\sigma}\right] \\
& =\mathbf{E}\left[Y_{\infty} \mid \mathcal{F}_{\sigma}\right] \\
& =Y_{\sigma} \quad \text { because } Y_{\infty}=Y_{\sigma} \quad \text { is } \mathcal{F}_{\sigma} \quad \text {-measurable } \\
& =0 \quad \text { since }\{\sigma<T\} \subset\{\sigma<\infty\} .
\end{aligned}
$$

This clearly shows that $\mathbb{P}[\{\sigma<T\}]=0$.

(2) On the set $\{T<\sigma\} \subset\{T<\infty\}$ we have that

$$
\begin{aligned}
0=X_{T} & =\mathbf{E}\left[X_{\infty} \mid \mathcal{F}_{T}\right] \\
& =\mathbf{E}\left[Y_{\infty} \mid \mathcal{F}_{T}\right] \\
& =\mathbf{E}\left[Y_{\sigma} \mid \mathcal{F}_{T}\right]
\end{aligned}
$$

We therefore obtain that $\int_{T<\sigma} Y_{\sigma} d \mathbb{P}=0$ and hence we have that $Y_{\sigma}=0$ on the set $\{T<\sigma\}$. From the martingale property for $\mathbb{Q}^{0}$ we then obtain that $\int_{T<\sigma} Y_{T} d \mathbb{Q}^{0}=0$. But this is clearly a contradiction to the definition of $\sigma$.

q.e.d. 
3.3 Corollary. Under the hypothesis and with the notation of lemma 3.2 we have that

(1) The jump of the martingale $Z_{t}^{\text {opt }}$ at the stopping time $T$ is zero, i.e., $Z_{t}^{\text {opt }}$ is continuous at $t=T$.

(2) The stopping time $T$ is announced by the sequence of stopping times

$$
T_{n}=\inf \left\{t \mid Z_{t}^{\mathrm{opt}} \leq \frac{1}{n}\right\} \wedge n
$$

Proof. The first claim follows from the fact that $T$ is predictable and from the martingale property. Indeed $\mathbf{E}\left[\Delta Z_{T}^{\text {opt }} \mid \mathcal{F}_{T-}\right]=0$. On the other hand the jump can only take non-positive values (as $Z_{T}=0$ while $Z_{t}>0$ for $t<T$ ), hence $\Delta Z_{T}^{\text {opt }}=0$ a.s.. The second claim follows trivially from the first claim.

The following lemma should be folklore, but for completeness we give a proof.

3.4 Lemma. If $U$ is a non-negative square integrable martingale, if $U_{0}>0$, if the stopping time $T=$ $\inf \left\{t \mid U_{t}=0\right\}$ is predictable and announced by a sequence of stopping times $\left(T_{n}\right)_{n \geq 1}$, then

$$
\mathbf{E}\left[\frac{U_{\infty}^{2}}{U_{T_{n}}^{2}} \mid \mathcal{F}_{T_{n}}\right] \rightarrow \infty
$$

on the set $\left\{U_{T}=0\right\}$.

Proof. Since the martingale is uniformly integrable, non-negative and since $U_{T_{n}}>0$, we find by the Cauchy-Schwarz inequality that

$$
\begin{aligned}
\mathbb{I} & =\mathbf{E}\left[\frac{U_{\infty}}{U_{T_{n}}} \mid \mathcal{F}_{T_{n}}\right] \\
& =\mathbf{E}\left[\frac{U_{\infty}}{U_{T_{n}}} \mathbb{I}\left\{U_{T} \neq 0\right\} \mid \mathcal{F}_{T_{n}}\right] \\
& \leq \mathbf{E}\left[\left(\frac{U_{\infty}}{U_{T_{n}}}\right)^{2} \mid \mathcal{F}_{T_{n}}\right]^{1 / 2} \mathbf{E}\left[\mathbb{I}\left\{U_{T} \neq 0\right\} \mid \mathcal{F}_{T_{n}}\right]^{1 / 2}
\end{aligned}
$$

Since $\mathbf{E}\left[\mathbb{I}\left\{U_{T} \neq 0\right\} \mid \mathcal{F}_{T_{n}}\right]$ tends to zero on the set $\left\{U_{T}=0\right\}$, the proof of the lemma is completed.

q.e.d.

We are now ready to prove the main theorem of this paper which has been stated in the introduction:

1.3 Main Theorem. Let $S$ be a continuous, $\mathbb{R}^{d}$-valued semi-martingale and suppose that $\mathcal{M}^{e}(\mathbb{P}) \cap$ $L^{2}(\mathbb{P}) \neq \emptyset$, i.e., there is at least one equivalent local martingale measure with square-integrable density. Then the variance-optimal measure $\mathbb{Q}^{\text {opt }}$ is a probability measure equivalent to $\mathbb{P}$.

Proof. We use the notation introduced above. Suppose that $\mathbb{P}\left[X_{T}=0\right]>\alpha>0$. The stopping time $T$ is predictable and is announced by the sequence $\left(T_{n}\right)_{n \geq 1}$. Because the martingale $Z^{0}$ is strictly positive it is uniformly bounded away from zero a.s., i.e. $\mathbb{P}\left[\inf _{0 \leq t} Z_{t}^{0}>0\right]=1$. Since the martingale $Z^{0}$ is also bounded in $L^{2}(\mathbb{P})$ we have that $\sup _{0 \leq t} \mathbf{E}\left[\left(Z_{\infty}^{0}\right)^{2} \mid \mathcal{F}_{t}\right]<\infty$ a.s.. On the other hand, the previous lemma shows that the expression

$$
\frac{\mathbf{E}\left[\left(Z_{\infty}^{\mathrm{opt}}\right)^{2} \mid \mathcal{F}_{T_{n}}\right]}{\left(Z_{T_{n}}^{\mathrm{opt}}\right)^{2}}
$$


tends to $\infty$ on the set $\left\{Z_{T}^{\text {opt }}=0\right\}$. It follows that for $n$ large enough the set

$$
A=\left\{\sup _{0 \leq t} \frac{\mathbf{E}\left[\left(Z_{\infty}^{0}\right)^{2} \mid \mathcal{F}_{t}\right]}{\left(Z_{t}^{0}\right)^{2}}<\frac{\mathbf{E}\left[\left(Z_{\infty}^{\circ p t}\right)^{2} \mid \mathcal{F}_{T_{n}}\right]}{\left(Z_{T_{n}}^{\circ p t}\right)^{2}}\right\}
$$

is non empty. As a consequence, for large enough $n$, the set

$$
A_{n}=\left\{\frac{\mathbf{E}\left[\left(Z_{\infty}^{0}\right)^{2} \mid \mathcal{F}_{T_{n}}\right]}{\left(Z_{T_{n}}^{0}\right)^{2}}<\frac{\mathbf{E}\left[\left(Z_{\infty}^{o p t}\right)^{2} \mid \mathcal{F}_{T_{n}}\right]}{\left(Z_{T_{n}}^{\text {opt }}\right)^{2}}\right\}
$$

is a non-empty set in $\mathcal{F}_{T_{n}}$. The martingale

$$
\begin{aligned}
Z_{t} & =Z_{t}^{\text {opt }} \text { for } t<T_{n} \\
& =\frac{Z_{t}^{0}}{Z_{T_{n}}^{0}} Z_{T_{n}}^{\text {opt }} \text { for } t \geq T_{n} \text { on the set } A_{n} \\
& =Z_{t}^{\text {opt }} \text { for } t \geq T_{n} \text { outside the set } A_{n}
\end{aligned}
$$

defines an equivalent martingale measure $\mathbb{Q}, d \mathbb{Q}=Z_{\infty} d \mathbb{P}$ with density $Z_{\infty}$ in $L^{2}(\mathbb{P})$. Because $\left\|Z_{\infty}\right\|_{2}<$ $\left\|Z_{\infty}^{o p t}\right\|_{2}$ we arrive at a contradiction.

q.e.d.

\section{Approximation of Continuous Processes}

In this section we apply the main theorem to a very natural and basic problem in Mathematical Finance, which was pointed out to us by H. Föllmer some years ago.

4.1 Problem. Given a continuous time stochastic process $\left(S_{t}\right)_{t \in \mathbb{R}_{+}}$based on and adapted to the structure $\left(\Omega, \mathcal{F},\left(\mathcal{F}_{t}\right)_{t \in \mathbb{R}_{+}}, \mathbb{P}\right)$ (satisfying suitable assumptions), find a sequence $\left(S_{t}^{n}\right)_{t \in \mathbb{R}_{+}}$of processes based on and adapted to $\Omega, \mathcal{F}^{n},\left(\mathcal{F}_{t}^{n}\right)_{t \in \mathbb{R}_{+}}, \mathbb{P}$ with the following properties.

(i) Each $S^{n}$ is finite, in the sense that $S^{n}$ is adapted to $\left(\Omega, \mathcal{F}^{n},\left(\mathcal{F}_{t}^{n}\right)_{t \in \mathbb{R}_{+}}\right)$where $\mathcal{F}^{n}$ and $\mathcal{F}_{t}^{n}$ are finite sub- $\sigma$-algebras of $\mathcal{F}$ and $\mathcal{F}_{t}$ respectively.

(ii) $S^{n}$ as well as $\left(\mathcal{F}^{n},\left(\mathcal{F}_{t}^{n}\right)_{t \in \mathbb{R}_{+}}\right)$converge in some reasonable sense to $S$ and $\left(\mathcal{F},\left(\mathcal{F}_{t}\right)_{t \in \mathbb{R}_{+}}\right)$.

(iii)For each $n$ there is a - in some sense naturally chosen - measure $\mathbb{Q}^{n}$ on $\mathcal{F}^{n}$ equivalent to the restriction of $\mathbb{P}$ to $\mathcal{F}^{n}$ such that there are only two possibilities: either $\left(\mathbb{Q}^{n}\right)_{n=1}^{\infty}$ converges, in which case it converges to an equivalent measure $\mathbb{Q}$ on $\mathcal{F}$ under which $S$ is a local martingale or $\left(\mathbb{Q}^{n}\right)_{n=1}^{\infty}$ diverges which implies that there is no equivalent local martingale measure for $S$ on $\mathcal{F}$.

There is an obvious interest in finding reasonable solutions to this problem of discrete approximation, which we deliberately formulated in somewhat vague terms. For example, we might think of a process $S$ with stochastic volatility which we want to approximate by discretisations modelled on finite trees. We shall not elaborate on particular examples but rather present a general methodology.

Of course, there is much known and a huge literature on the aspects (i) and (ii) of the above problem. The new ingredient is the aspect (iii) pertaining to the construction of equivalent martingale measures, which is of central importance in Mathematical Finance. The problem pertains in particular to the question in which "natural sense" the martingale measures $\mathbb{Q}^{n}$ should be chosen for the finite processes $S^{n}$.

Let us start with the easy situation of a complete market, i.e., if the process $S$ admits exactly one equivalent local martingale measure $\mathbb{Q}$ on $\mathcal{F}$. In this case the problem of "natural choice" does not arise and it is standard to approximate $S$ by a sequence of complete discretisations $S^{n}$, i.e., such that there is exactly one equivalent martingale measure $\mathbb{Q}^{n}$ on $\mathcal{F}^{n}$ and such that $\mathbb{Q}^{n}$ converges to $\mathbb{Q}$ (in a sense 
to be specified). For example, we have the well known approximation of Brownian motion by binomial processes.

The fun in problem 4.1 starts if we pass to non-complete markets where the problem of "natural choice" becomes crucial. For example, choosing for each $n \in \mathbb{N}$ the minimal local martingale measure $\mathbb{Q}^{n}$ on $\mathcal{F}^{n}$ may turn out to be a poor choice: the limit measure $\mathbb{Q}$ should - in any reasonable construction - again be the minimal local martingale measure; but the examples in [S 93] and [DS 94] show that — even if $S$ is a very nicely behaved process - the minimal martingale measure need not exist. In other words, the minimal martingale measure may fail to be the target, to which the $\mathbb{Q}^{n}$ can aim to converge to.

On the other hand, the main theorem 1.3 above gives us a possible target for the $\mathbb{Q}^{n}$ to aim for, namely the variance-optimal measure. We shall present a possible construction responding to problem 4.1 in the following situation. We assume $S=\left(S_{t}\right)_{t \in \mathbb{R}_{+}}$to be a continuous semi-martingale, which we also assume to be one-dimensional. We shall add some technical assumptions as we proceed in our construction. For the moment, we only suppose that $S$ is based on $\left(\Omega, \mathcal{F},\left(\mathcal{F}_{t}\right)_{t \in \mathbb{R}_{+}}, \mathbb{P}\right)$ satisfying the usual assumptions and such that $\mathcal{F}_{0}$ consists of the null-sets and their complements only and $S_{0}=0$. We also assume that the process $S$ "never runs out of steam", i.e.

$$
\lim _{t \rightarrow \infty}\langle S\rangle_{t}=\infty \quad \text { a.s. }
$$

This assumption will be convenient for the time-change arguments below; it is easy to convince oneself that this assumption is not really a restriction of generality.

4.2 Theorem. Let $\left(S_{t}\right)_{t \in \mathbb{R}_{+}}$be a one-dimensional continuous semi-martingale based on $\left(\Omega, \mathcal{F},\left(\mathcal{F}_{t}\right)_{t \in \mathbb{R}}, \mathbb{P}\right)$ such that $\langle S\rangle_{t} \rightarrow \infty$ almost surely. Define

$$
T_{u}=\inf \left\{t:\langle S\rangle_{t} \geq u\right\}
$$

and denote by $\left(R_{u}\right)_{u \in \mathbb{R}_{+}}$the time-changed process

$$
R_{u}=S_{T_{u}}
$$

and by $\left(\mathcal{G}_{u}\right)_{u \in \mathbb{R}_{+}}$the natural filtration generated by $\left(R_{u}\right)_{u \in \mathbb{R}_{+}}$so that $\mathcal{G}_{u} \subseteq \mathcal{F}_{T_{u}}$ and $\mathcal{G}=\sigma\left(\left(\mathcal{G}_{u}\right)_{u \in \mathbb{R}}\right) \subseteq$ $\mathcal{F}$.

(a) If there is an equivalent local martingale measure $\mathbb{Q}^{0}$ for the process $R_{u}$ on $\mathcal{G}$, then under $\mathbb{Q}^{0}$ the process $R_{u}$ is a standard Brownian motion with respect to its natural filtration $\left(\mathcal{G}_{u}\right)_{u \in \mathbb{R}_{+}}$. The Doob-Meyer decomposition (with respect to $\mathbb{P}$ and the filtration $\left(\mathcal{G}_{u}\right)$ ) of $R_{u}$ is of the form

$$
d R_{u}=d M_{u}+\alpha_{u} d\langle M\rangle_{u}=d M_{u}+\alpha_{u} d u
$$

where $\left(M_{u}\right)_{u \in \mathbb{R}_{+}}$is a standard Brownian motion with respect to $\mathbb{P}$ and to the filtration $\left(\mathcal{G}_{u}\right)_{u \in \mathbb{R}_{+}}$and $\alpha$ is a $\left(\mathcal{G}_{u}\right)$-predictable process with $\int_{0}^{\infty}\left|\alpha_{u}\right| d u<\infty$ almost surely. In this case the measure $\mathbb{Q}^{0}$ on $\mathcal{G}$ is the unique local martingale measure for $R_{u}$ and its density is given by

$$
\frac{d \mathbb{Q}^{0}}{d \mathbb{P}}=\mathcal{E}(-\alpha \cdot M)_{\infty}
$$

Furthermore $R_{u}$ is a martingale (and not only a local martingale) under $\mathbb{Q}^{0}$.

(b) If the process $S_{t}$ admits an equivalent local martingale measure $\mathbb{Q}$ on $\mathcal{F}$, then the restriction of $\mathbb{Q}$ to $\mathcal{G}$ coincides with the above defined unique local martingale measure $\mathbb{Q}^{0}$ for $R_{u}$.

Proof. (a) is rather obvious and (b) results from the fact that each simple stochastic integral on $R$ (with respect to the filtration $\mathcal{G}_{u}$ ) may be written as a simple stochastic integral on $S$ (with respect to the filtration $\mathcal{F}_{t}$ ).

q.e.d. 
The theorem suggests the following strategy to analyse the set $\mathcal{M}^{e}(\mathbb{P})$ of equivalent local martingale measures for the process $S$ on $\mathcal{F}$. First we pass to the time change $R_{u}$ of $S_{t}$ and check whether the (unique) martingale measure $\mathbb{Q}^{0}$ for $R$ exists on $\mathcal{G}$. This should be (relatively) easy to check as there is a formula at hand. The existence of $\mathbb{Q}^{0}$ is a necessary condition for the existence of a local martingale measure $\mathbb{Q}$ for $S$ on $\mathcal{F}$. As a second step one has to analyze, whether (and in which possible ways) $\mathbb{Q}^{0}$ may be extended from $\mathcal{G}$ to $\mathcal{F}$ by maintaining the property that $\mathbb{Q}$ is a local martingale measure for $S$ with respect to the filtration $\mathcal{F}_{t}$.

To study the enlargements of the filtration $\left(\mathcal{G}_{u}\right)_{u \in \mathbb{R}_{+}}$which are contained in the filtration $\left(\mathcal{F}_{T_{u}}\right)_{u \in \mathbb{R}_{+}}$we introduce a somewhat formal concept.

4.3 Definition. Let $\mathcal{A}$ denote the family of all objects $A$ of the form

$$
A=\left(u_{1}, \ldots, u_{n}, \mathcal{H}_{u_{1}}, \ldots, \mathcal{H}_{u_{n}}\right)
$$

where $n \in \mathbb{N}, 0<u_{1}<\cdots<u_{n}$ and $\mathcal{H}_{u_{i}}$ are finite sub- $\sigma$-algebras of $\mathcal{F}_{T_{u_{i}}}$ such that $\left(\mathcal{H}_{u_{i}}\right)_{i=1}^{n}$ is increasing. We sometimes denote $u_{0}=0, \mathcal{H}_{u_{0}}=\{\emptyset, \Omega\}$ and $u_{n+1}=\infty$. On the family $\mathcal{A}$ we define a partial order by saying that

$$
B=\left(v_{1}, \ldots, v_{m}, \mathcal{K}_{v_{1}}, \ldots, \mathcal{K}_{v_{m}}\right)
$$

is bigger than $A$ if $\left\{v_{1}, \ldots, v_{m}\right\}$ contains $\left\{u_{1}, \ldots, u_{n}\right\}$ and $v_{j}=u_{i}$ implies that $\mathcal{K}_{v_{j}} \supseteq \mathcal{H}_{u_{i}}$.

For $A \in \mathcal{A}$ we define the filtration $\left(\mathcal{G}_{u}^{A}\right)_{u \in \mathbb{R}_{+}}$by

$$
\mathcal{G}_{u}^{A}=\sigma\left(\mathcal{G}_{u}, \mathcal{H}_{u_{i}} \mid u_{i} \leq u\right),
$$

and the $\sigma$-algebra $\mathcal{G}^{A}$ by

$$
\mathcal{G}^{A}=\sigma\left(\mathcal{G}, \mathcal{H}_{u_{n}}\right)
$$

It is intuitively obvious that the family of filtrations $\left(\mathcal{G}_{u}^{A}\right)_{A \in \mathcal{A}}$ converges to the filtration $\left(\mathcal{F}_{T_{u}}\right)_{u \in \mathbb{R}_{+}}$. To make this statement precise we adopt the usual $L^{2}$-setting of this paper. It will be convenient to add a mild technical assumption.

General Assumption: For the rest of this section we assume that $S$ is a one-dimensional continuous semi-martingale, $\langle S\rangle_{t} \rightarrow \infty$ a.s., and that, for each $u_{0} \in R_{+}, R_{u_{0}}^{*}=\sup _{0 \leq u \leq u_{0}}\left|R_{u}\right| \in L^{p}(\mathbb{P})$ for some $p>2$. We shall also assume that the martingale measure $\mathbb{Q}^{0}$ for the process $\left(R_{u}\right)_{u \in \mathbb{R}_{+}}$with respect to the filtration $\left(\mathcal{G}_{u}\right)_{u \in \mathbb{R}_{+}}$exists and is equivalent to $\mathbb{P}$ (on the $\sigma$-algebra $\mathcal{G}$ ).

4.4 Proposition. Under the above assumption let $f=(H \cdot S)_{\infty}$ be an element of $K_{0}$, i.e., a simple integral on $S$ of the form introduced in 1.1 above (with respect to the filtration $\left(\mathcal{F}_{t}\right)_{t \in \mathbb{R}_{+}}$).

For $\varepsilon>0$, there is $A \in \mathcal{A}$ and a simple integrand $H^{A}$ with respect to $\left(R_{u}\right)_{u \in \mathbb{R}_{+}}$and the filtration $\left(\mathcal{G}_{u}^{A}\right)$ such that, for $f^{A}=\left(H^{A} \cdot R\right)_{\infty}$, we have that

$$
\left\|f^{A}-f\right\|_{L^{2}(\mathbb{P})}<\varepsilon
$$

Proof. We may suppose that

$$
f=h\left(S_{T(2)}-S_{T(1)}\right)
$$

where $T^{(1)} \leq T^{(2)}$ are stopping times such that the stopped process $S^{T^{(2)}}$ is bounded and $h$ is a bounded $\mathcal{F}_{T^{(1)}}$-measurable function. We may also suppose that $h$ is a simple function and that $T^{(2)}$ is bounded by some $T_{u}$, say $T^{(2)} \leq T_{M}$ for some $M \in \mathbb{R}_{+}$. Indeed, for the last assertion note that $\left(T_{u}\right)_{u \in \mathbb{R}_{+}}$increases to infinity, hence $\left(S_{T_{u} \wedge T^{(1)}}-S_{T^{(1)}}\right)_{u \in \mathbb{R}_{+}}$as well as $\left(S_{T_{u} \wedge T^{(2)}}-S_{T^{(2)}}\right)_{u \in \mathbb{R}_{+}}$tend to zero almost surely as $u \rightarrow \infty$. As they also remain uniformly bounded they also converge to zero in $L^{2}(\mathbb{P})$. 
By writing $f=h\left(S_{T^{(2)}}-S_{T_{M}}\right)+h\left(S_{T_{M}}-S_{T^{(1)}}\right)$ we see that we even may assume that $T^{(2)}$ equals $T_{M}$.

Let $0<u_{1}<\cdots<u_{n}=M$, and define $\mathcal{H}_{u_{i}}$ inductively, for $i=1, \ldots, n$, to be generated by $\mathcal{H}_{u_{i-1}}$, $\left\{T^{(1)} \leq T_{u_{i}}\right\}$, and $h \mathbb{I}\left\{T^{(1)} \leq T_{u_{i}}\right\}$. Let $A=\left(u_{1}, \ldots, u_{n}, \mathcal{H}_{u_{1}}, \ldots \mathcal{H}_{u_{n}}\right)$ and define the random variable

$$
\begin{aligned}
f^{A} & =\sum_{i=1}^{n} h \mathbb{I}\left\{T^{(1)} \leq T_{u_{i-1}}\right\}\left(S_{T_{u_{i}}}-S_{T_{u_{i-1}}}\right) \\
& =\sum_{i=1}^{n} h \mathbb{I}\left\{T^{(1)} \leq T_{u_{i-1}}\right\}\left(R_{u_{i}}-R_{u_{i-1}}\right)
\end{aligned}
$$

which is a simple stochastic integral on $R$ with respect to the filtration $\left(\mathcal{G}_{u}^{A}\right)_{u \in \mathbb{R}_{+}}$.

Note that our technical assumption implies that the random variables $f^{A}$ remain bounded in $L^{p}(\mathbb{P})$; if $\left(A^{j}\right)_{j=1}^{\infty}$ is a sequence in $\mathcal{A}, A^{j}=\left(u_{1}^{j}, \ldots, u_{n_{j}}^{j}, \mathcal{H}_{u_{1}}^{j}, \ldots \mathcal{H}_{u_{n_{j}}}^{j}\right)$ constructed as above such that $\lim _{j \rightarrow \infty} \max _{1 \leq i \leq n_{j}} \mid u_{i}^{j}-$ $u_{i-1}^{j} l=0$, it follows from the continuity of $S$ that $\left(f^{A^{j}}\right)_{j=1}^{\infty}$ converges almost surely to $f$. Therefore $f^{A_{j}}$ converges to $f$ with respect to the norm of $L^{2}(\mathbb{P})$, which finishes the proof.

q.e.d.

We may reformulate the assertion of Proposition 4.4 in the following way. Identifying $L^{2}\left(\Omega, \mathcal{G}^{A}, \mathbb{P}\right)$ with a subspace of $L^{2}(\Omega, \mathcal{F}, \mathbb{P})$ and denoting by $K_{0}^{A}$ the space of simple stochastic integrals on $R_{u}$ with respect to the filtration $\left(\mathcal{G}_{u}^{A}\right)$, the assertion of proposition 4.4 then becomes tantamount to saying that $\bigcup_{A \in \mathcal{A}} K_{0}^{A}$ is a $\|\cdot\|_{2}$-dense subspace of $K_{0}$.

As a next step we analyze in detail the possible martingale measure extensions of the measure $\mathbb{Q}^{0}$ on $\mathcal{G}$ to a martingale measure $\mathbb{Q}^{A}$ on $\mathcal{G}^{A}$. In order to do the book-keeping of the subsequent proposition 4.5 we introduce some notation. We denote by atom $(\mathcal{H})$ the atoms of a finite $\sigma$-algebra $\mathcal{H}$, i.e., the elements of $\mathcal{H}$ which contain only $\emptyset$ as a proper subset. If $\mathcal{H}_{1} \subseteq \mathcal{H}_{2}$ are both finite $\sigma$-algebras and $I$ is an atom of $\mathcal{H}_{1}$ we denote - if no confusion can arise - by atom $(I)$ the atoms of $\mathcal{H}_{2}$ contained in $I$. If $\mathcal{H}_{0} \subseteq \cdots \subseteq \mathcal{H}_{n}$ are increasing finite $\sigma$-algebras of $0 \leq k \leq j \leq n$ and $I$ an atom of $\mathcal{H}_{j}$, then we denote by $\pi_{k}(I)$ the unique atom of $\mathcal{H}_{k}$ which contains $I$. The reader may want to consult example 2.5 as an easy illustration of the situation described by the subsequent result.

4.5 Proposition. Under the above assumption let

$$
A=\left(u_{1}, \ldots, u_{n}, \mathcal{H}_{u_{1}}, \ldots, \mathcal{H}_{u_{n}}\right) \in \mathcal{A}
$$

be given. There is a one-to-one correspondence between

(i) the extensions $\mathbb{Q}$ of $\mathbb{Q}^{0}$ to the $\sigma$-algebra $\mathcal{G}^{A}$ such that $\mathbb{Q}$ is an equivalent local martingale measure for $R_{u}$ with respect to the filtration $\mathcal{G}_{u}^{A}$.

(ii) The families of functions $\left(\left(f_{i}^{I_{i}}\right)_{I_{i} \in \operatorname{atom}\left(\mathcal{H}_{u_{i}}\right)}\right)$ with the following properties:

(a) each $f_{i}^{I_{i}}$ is measurable with respect to the $\sigma$-algebra $\mathcal{G}_{u_{i}-}^{A}$ and takes values a.s. in $\left.] 0,1\right]$ on the support of $\mathbf{E}\left[\mathbb{1} I_{i} \mid \mathcal{G}_{u_{i}-}^{A}\right]$ and zero elsewhere.

(b) For each $1 \leq i \leq n$ and each atom $I_{i-1} \in \mathcal{H}_{u_{i-1}}$ we have that

$$
\sum_{I_{i} \in \operatorname{atom}\left(I_{i-1}\right)} f_{i}^{I_{i}}=\mathbb{I} I_{i-1} \text { a.s. }
$$


The correspondence between (i) and (ii) is given by the subsequent formula for the density $Z=\frac{d \mathbb{Q}}{d \mathbb{P}}$ of the measure $\mathbb{Q}$ on $\mathcal{G}^{A}$

$$
Z_{\infty}(\omega)=\sum_{I_{n} \in \operatorname{atom}\left(\mathcal{H}_{u_{n}}\right)} Z_{\infty}^{0}(\omega) \cdot \prod_{i=1}^{n}\left(g_{i}^{\pi_{i}\left(I_{n}\right)}(\omega) \mathbb{I} I_{n}(\omega)\right)
$$

where

$$
g_{i}^{I_{i}}=\frac{f_{i}^{I_{i}}}{\mathbf{E}\left[\mathbb{I} I_{i} \mid \mathcal{G}_{u_{i}-}^{A}\right]}
$$

with the usual convention $\frac{0}{0}=0$.

Remark. We may interpret, for given $0 \leq i \leq n$ and $I_{i-1} \in \mathcal{H}_{u_{i-1}}$, the family of functions $\left(f_{i}^{I_{i}}\right)_{I_{i} \in \operatorname{atom}\left(I_{i-1}\right)}$ as the rule of distributing the mass of the probability measure $\mathbb{Q}$ on $I_{i-1}$ among the atoms $I_{i} \in$ atom $\left(I_{i-1}\right)$. The assertion of proposition 4.5 means that we obtain the general form of a local martingale measure extension $\mathbb{Q}$ to $\mathcal{G}^{A}$ iff this distribution of weights is done in a $\sigma\left(\mathcal{G}_{u_{i}}, \mathcal{H}_{u_{0}}, \ldots, \mathcal{H}_{u_{i-1}}\right)$-measurable (but otherwise arbitrary) way assigning to each $I_{i}$ strictly positive mass.

Proof. The verification of the assertion of the proposition is mainly a matter of book-keeping.

Let $\mathbb{Q}$ be a local martingale measure for $R_{u}$ on $\mathcal{G}^{A}$ with respect to the filtration $\mathcal{G}_{u}^{A}$. Denote by $\left(Z_{u}\right)_{u \in \mathbb{R}_{+}}$ the corresponding density process. For $1 \leq i \leq n$ and an atom $I_{i} \in \mathcal{H}_{u_{i}}$ define

$$
f_{i}^{I_{i}}=\frac{\mathbf{E}\left[Z_{u_{i}} \Pi I_{i} \mid \mathcal{G}_{u_{i}-}^{A}\right]}{Z_{u_{i}-}}
$$

The verification of properties (ii) (a) and (b) is straightforward. To verify that $Z$ is indeed of the form given by formula (4.1) denote by $Z$ the density process of $\mathbb{Q}$ with respect to the filtration $\left(\mathcal{G}_{u}^{A}\right)_{u \in \mathbb{R}_{+}}$and by $\tilde{Z}$ the $\mathcal{G}_{u}^{A}$-martingale given by taking conditional expectations in (4.1), so that, for $j=1, \ldots, n+1$ and $t \in\left[u_{j-1}, u_{j}\right.$ [ we have

$$
\tilde{Z}_{t}=\sum_{I_{j-1} \in \operatorname{atom}\left(\mathcal{H}_{u_{j-1}}\right)} Z_{t}^{0} \prod_{i=1}^{j-1} g_{i}^{\pi_{i}\left(I_{j-1}\right)} \mathbb{I} I_{j-1} .
$$

Indeed, to verify (4.2) note that

$$
\begin{aligned}
\tilde{Z}_{u_{n}} & =\sum_{I_{n} \in \operatorname{atom}\left(\mathcal{H}_{u_{n}}\right)} Z_{u_{n}}^{0} \prod_{i=1}^{n} g_{i}^{\pi_{i}\left(I_{n}\right)} \mathbb{I} I_{n} \\
\tilde{Z}_{u_{n}-} & =Z_{u_{n}}^{0} \sum_{I_{n} \in \operatorname{atom}\left(\mathcal{H}_{u_{n}}\right)} \mathbf{E}\left[g_{n}^{I_{n}} \mathbb{I} I_{n} \mid \mathcal{G}_{u_{n}-}^{A}\right] \prod_{i=1}^{n-1} g_{i}^{\pi_{i}\left(I_{n}\right)} \\
& =Z_{u_{n}}^{0} \sum_{I_{n} \in \operatorname{atom}\left(\mathcal{H}_{u_{n}}\right)}\left(\frac{f_{n}^{I_{n}} \mathbf{E}\left[\mathbb{I} I_{n} \mid \mathcal{G}_{u_{n}-}^{A}\right]}{\mathbf{E}\left[\mathbb{I} I_{n} \mid \mathcal{G}_{u_{n}}^{A}\right]}\right) \prod_{i=1}^{n-1} g_{i}^{\pi_{i}\left(I_{n}\right)} \\
& =Z_{u_{n}}^{0} \sum_{I_{n-1} \in \operatorname{atom}\left(\mathcal{H}_{u_{n-1}}\right)} \prod_{i=1}^{n-1} g_{i}^{\pi_{i}\left(I_{n-1}\right)} \mathbb{I} I_{n-1} .
\end{aligned}
$$

Continuing in an obvious way for $i=n-1, \ldots, 0$ we verify (4.2).

To establish that $Z$ equals $\tilde{Z}$ we observe that

$$
\frac{Z_{u_{j}}}{Z_{u_{j}-}}=\frac{\tilde{Z}_{u_{j}}}{\tilde{Z}_{u_{j}-}}=\sum_{I_{j} \in \operatorname{atom} \mathcal{H}\left(u_{j}\right)} g_{j}^{I_{j}} \mathbb{I} I_{j}
$$


for $j=1, \ldots, n$ and

$$
\frac{Z_{u_{j}-}}{Z_{u_{j-1}}}=\frac{\tilde{Z}_{u_{j}-}}{\tilde{Z}_{u_{j-1}}}=\frac{Z_{u_{j-}-}^{0}}{Z_{u_{j-1}}^{0}}
$$

for $j=1, \ldots, n+1$. Equation (4.3) follows from the definition of $f_{i}^{I_{i}}$ and (4.4) from the uniqueness of the local martingale measure $\mathbb{Q}^{0}$ with respect to the filtration $\mathcal{G}_{u}^{A}$.

Summing up, we have shown that for each equivalent local martingale measure $\mathbb{Q}$ on $\mathcal{G}^{A}$ we may de-

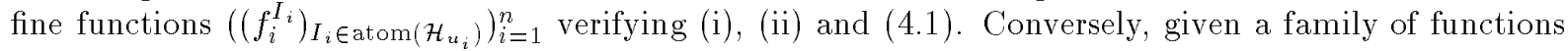
$\left(\left(f_{i}^{I_{i}}\right)_{I_{i} \in \operatorname{atom}}\left(\mathcal{H}_{u_{i}}\right)\right)_{i=1}^{n}$ verifying (i) and (ii), we may define $\mathbb{Q}$ via (4.1) and by going through the above identities again it follows that $\mathbb{Q}$ is a probability measure equivalent to $\mathbb{P}$ on $\mathcal{G}^{A}$, such that $R_{u}$ is a local martingale with respect to $\mathbb{Q}$ and the filtration $\left(\mathcal{G}_{u}^{A}\right)_{u \in \mathbb{R}_{+}}$. Note that the equivalence of $\mathbb{Q}$ to $\mathbb{P}$ follows from the fact that the functions $f_{i}^{I_{i}}$ are almost surely strictly positive on the support of $\mathbf{E}\left[\mathbb{1} I_{i} \mid \mathcal{G}_{u_{i}-}\right]$.

The explicit description of the possible equivalent local martingale extensions of $\mathbb{Q}^{0}$ to $\mathcal{G}^{A}$ in proposition 4.5 now allows us to obtain an explicit characterization of the "variance-optimal" extension.

We start with an elementary lemma.

4.6 Lemma. (a) Let $\left(a_{k}\right)_{k=1}^{N}$ be strictly positive real numbers. Then the minimization problem

$$
F\left(x_{1}, \ldots, x_{N}\right)=\sum_{k=1}^{N} x_{k}^{2} a_{k} \rightarrow \min !
$$

where we minimize over all real numbers $x_{1}, \ldots, x_{N}$ under the constraint

$$
\sum_{k=1}^{N} x_{k}=1
$$

has a unique solution, namely

$$
\hat{x}_{k}=\frac{a_{k}^{-1}}{\sum_{k=1}^{N} a_{k}^{-1}} .
$$

We have that $F\left(\hat{x}_{1}, \ldots, \hat{x}_{N}\right)=\left(\sum_{k=1}^{N} a_{k}^{-1}\right)^{-1}$.

(b) More generally, let $\left(a_{k}(\omega)\right)_{k=1}^{N}$ be strictly positive measurable functions, defined on some $(\Omega, \mathcal{F}, P)$. Then the minimization problem

$$
\mathbf{E}\left[\sum_{k=1}^{N} x_{k}^{2}(\omega) a_{k}(\omega)\right] \rightarrow \min !
$$

where we minimize over all real-valued measurable functions $x_{1}(\omega), \ldots, x_{N}(\omega)$ under the constraint

$$
\sum_{k=1}^{N} x_{k}(\omega) \equiv 1
$$

has a unique solution (unique up to equality almost everywhere), namely 


$$
\hat{x}_{k}(\omega)=\frac{a_{k}(\omega)^{-1}}{\sum_{k=1}^{N} a_{k}^{-1}(\omega)}
$$

Proof. (a) follows from elementary calculus with Lagrange multipliers. The second part is an almost immediate consequence of the first one by reasoning pointwise on $\omega \in \Omega$. Let $x_{k}(\omega)$ be defined as above and let $y_{k}(\omega)$ be any measurable real-valued functions satisfying the constraint

$$
\sum_{k=1}^{N} y_{k}(\omega) \equiv 1
$$

Then for each $\omega \in \Omega$ we have

$$
\sum_{k=1}^{N} x_{k}^{2}(\omega) a_{k}(\omega) \leq \sum_{k=1}^{N} y_{k}^{2}(\omega) a_{k}(\omega)
$$

with equality holding iff $x_{k}(\omega)=y_{k}(\omega)$, for each $k=1, \ldots, N$. The conclusion now follows.

Note that in lemma 4.6 we have in particular that, for the solution $\hat{x}_{1}, \ldots, \hat{x}_{n}$, each $\hat{x}_{k}$ is strictly positive. The lemma provides us with a formula of variance-optimal distribution of weights which allows us to

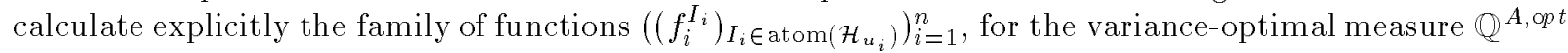
with respect to $R_{u}$ and the filtration $\mathcal{G}_{u}^{A}$. Let us show this in some detail.

Denoting by $Z^{A, \text { opt }}$ the density process associated to $\mathbb{Q}^{A, \text { opt }}$ we shall determine $Z^{A, \text { opt }}$ by backward induction on $i=n, \ldots, 1$. First note that

$$
\frac{Z_{t}^{A, o p t}}{Z_{u_{n}}^{A, \circ p t}}=\frac{Z_{t}^{0}}{Z_{u_{n}}^{0}} \quad \text { for } t \in\left[u_{n}, \infty\right]
$$

Indeed, this follows from the fact that any local martingale measure $\mathbb{Q}$ on $\mathcal{G}^{A}$ for $R-R^{u_{n}}$ is uniquely determined by its restriction to $\mathcal{G}_{u_{n}}^{A}$.

The subtle point consists in calculating the (possible) jumps of $Z^{A, o p t}$ at time $u_{n}$. To do so, denote, for $I_{n} \in \operatorname{atom}\left(\mathcal{H}_{u_{n}}\right)$, the $\mathcal{G}_{u_{n}-}^{A}$-measurable functions

$$
\begin{aligned}
a_{n}^{I_{n}} & =\frac{\mathbf{E}\left[\left(\frac{Z_{\infty}^{A, \text { opt }}}{Z_{u_{n}}^{A, \text { opt }}}\right)^{2} \mathbb{I} I_{n} \mid \mathcal{G}_{u_{n}}^{A}-\right]}{\mathbf{E}\left[\mathbb{I} I_{n} \mid \mathcal{G}_{u_{n}-}^{A}\right]^{2}} \\
& =\frac{\mathbf{E}\left[\left(\frac{Z_{\infty}^{0}}{Z_{u_{n}}^{0}}\right)^{2} \mathbb{I} I_{n} \mid \mathcal{G}_{u_{n}-}^{A}\right]}{\mathbf{E}\left[\mathbb{I} I_{n} \mid \mathcal{G}_{u_{n}-}^{A}\right]^{2}} .
\end{aligned}
$$

To construct the functions $\left(\hat{f}_{n}^{I_{n}}\right)_{I_{n} \in \operatorname{atom}\left(\mathcal{H}_{u_{n}}\right)}$ corresponding to $\mathbb{Q}^{A, \text { opt }}$ via proposition 4.5 let

$$
\hat{f}_{n}^{I_{n}}=\frac{\left(a_{n}^{I_{n}}\right)^{-1}}{\sum_{I \in \operatorname{atom}\left(\pi_{n-1}\left(I_{n}\right)\right)}\left(a_{n}^{I}\right)^{-1}} \mathbb{I} \pi_{n-1}\left(I_{n}\right)
$$


and

$$
\hat{g}_{n}^{I_{n}}=\frac{\hat{f}_{n}^{I_{n}}}{\mathbf{E}\left[\Pi I_{n} \mid \mathcal{G}_{u_{n}-}^{A}\right]}
$$

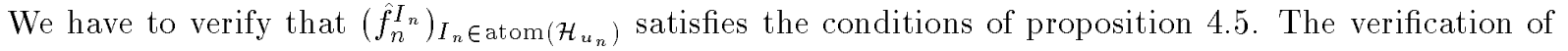
a) and b) of assertion ii) is straightforward. For example, note that

$$
\begin{aligned}
\sum_{I_{n} \in \operatorname{atom}\left(I_{n-1}\right)} \hat{f}_{n}^{I_{n}} & =\frac{\sum_{I_{n} \in \operatorname{atom}\left(I_{n-1}\right)}\left(a_{n}^{I_{n}}\right)^{-1}}{\sum_{I_{n} \in \operatorname{atom}\left(I_{n-1}\right)}\left(a_{n}^{I_{n}}\right)^{-1}} \cdot \mathbb{I} I_{n-1} \\
& =\mathbb{I} I_{n-1} .
\end{aligned}
$$

We claim that — given the function $Z_{u_{n}-}^{A, o p t}$ - the formula

$$
\frac{Z_{u_{n}}^{A, \circ p t}}{Z_{u_{n}-\mathrm{opt}}^{A,}}=\sum_{I_{n} \in \operatorname{atom}\left(\mathcal{H}_{u_{n}}\right)} \hat{g}_{n}^{I_{n}} \Pi I_{n}
$$

minimizes the quantity $\left\|Z_{\infty}\right\|_{L^{2}(\mathbb{P})}^{2}$ over all local martingale densities $Z_{\infty}$ with $Z_{u_{n}-}=Z_{u_{n}-}^{A, o p t}$. Indeed, we have to solve the optimization problem

$$
\mathbf{E}\left[\left(\frac{Z_{\infty}}{Z_{u_{n}-o p t}^{A}}\right)^{2}\right] \rightarrow \operatorname{min!}
$$

where we minimize over all densities $Z_{\infty}$ obtained via functions $\left(f_{n}^{I_{n}}\right)_{I_{n} \in \operatorname{atom}\left(\mathcal{H}_{u_{n}}\right)}\left(\operatorname{resp} .\left(g_{n}^{I_{n}}\right)_{I_{n} \in \operatorname{atom}\left(\mathcal{H}_{u_{n}}\right)}\right)$ as described in proposition 4.5. Noting that an atom $I_{n-1} \in \operatorname{atom}\left(\mathcal{H}_{u_{n-1}}\right)$ is $\mathcal{G}_{u_{n}-}^{A}$-measurable, we may argue on each $I_{n-1} \in \operatorname{atom}\left(\mathcal{H}_{u_{n-1}}\right)$ separately so that in order to verify $(4.5)$ we have to show that $Z_{\infty}^{A, o p t}$ solves the problem

$$
\mathbf{E}\left[\left(\frac{Z_{\infty}}{Z_{u_{n}-}^{A, o p t}}\right)^{2} \mathbb{I} I_{n-1}\right] \rightarrow \operatorname{min!} \quad \text { for } I_{n-1} \in \operatorname{atom}\left(\mathcal{H}_{u_{n-1}}\right)
$$

Using the equations

$$
\begin{aligned}
& Z_{u_{n}} \Pi I_{n-1}=Z_{u_{n}-}^{A, o p t} . \sum_{I_{n} \in \operatorname{atom}\left(I_{n-1}\right)} g_{n}^{I_{n}} \mathbb{I} I_{n} \\
& =Z_{u_{n}-}^{A, \circ p t} \cdot \sum_{I_{n} \in \operatorname{atom}\left(I_{n-1}\right)} \frac{f_{n}^{I_{n}}}{\mathbf{E}\left[\Pi I_{n} \mid \mathcal{G}_{u_{n}-}^{A}\right]} \mathbb{I} I_{n}
\end{aligned}
$$

and

$$
\frac{Z_{\infty}}{Z_{u_{n}}}=\frac{Z_{\infty}^{0}}{Z_{u_{n}}^{0}}
$$

we may calculate 


$$
\begin{aligned}
\mathbf{E}\left[\left(\frac{Z_{\infty}}{Z_{u_{n}-}^{A, \text { opt }}}\right)^{2} \mathbb{I} I_{n-1}\right] & =\mathbf{E}\left[\sum_{I_{n} \in \operatorname{atom}\left(I_{n-1}\right)}\left(\frac{Z_{\infty}}{Z_{u_{n}-}^{A, o p t}}\right)^{2} \mathbb{I} I_{n}\right] \\
& =\mathbf{E}\left[\sum_{I_{n} \in \operatorname{atom}\left(I_{n-1}\right)}\left(\frac{Z_{\infty}^{0}}{Z_{u_{n}}^{0}}\right)^{2} \cdot\left(\frac{\left(f_{n}^{I_{n}}\right)}{\mathbf{E}\left[\mathbb{I I} I_{n} \mid \mathcal{G}_{u_{n}-}^{A}\right]}\right)^{2} \mathbb{I} I_{n}\right] \\
& =\mathbf{E}\left[\sum_{I_{n} \in \operatorname{atom}\left(I_{n-1}\right)} \frac{\left(f_{n}^{I_{n}}\right)^{2}}{\mathbf{E}\left[\mathbb{I} I_{n} \mid \mathcal{G}_{u_{n}-}^{A}\right]^{2}} \cdot \mathbf{E}\left[\left(\frac{Z_{\infty}^{0}}{Z_{u_{n}}^{0}}\right)^{2} \mathbb{I} I_{n} \mid \mathcal{G}_{u_{n}-}^{A}\right]\right] \\
& =\mathbf{E}\left[\sum_{I_{n} \in \operatorname{atom}\left(I_{n-1}\right)}\left(f_{n}^{I_{n}}\right)^{2} \cdot a_{n}^{I_{n}}\right] .
\end{aligned}
$$

Noting the constraint $\sum_{I_{n} \in \operatorname{atom}\left(I_{n-1}\right)} f_{n}^{I_{n}}=\mathbb{I} I_{n-1}$ we are exactly in the situation of lemma 4.6 (b) which allows us to conclude that - whatever $Z_{u_{n}-}^{A \text {,opt }}$ may be - the use of $\left(\hat{f}_{n}^{I_{n}}\right)_{I_{n} \in \operatorname{atom}\left(\mathcal{H}_{u_{n}}\right)}$ is the optimal choice to extend $Z_{u_{n}-}^{A, \text { opt }}$ to $Z_{u_{n}}^{A, \text { opt }}$ and therefore to $Z_{\infty}^{A, \text { opt }}$.

Now we may continue by backward induction to calculate $Z_{u}^{A, o p t}$. By the uniqueness of $\mathbb{Q}^{0}$ with respect to $\mathcal{G}_{u}$ there is no problem to calculate the ratio of $Z_{u}^{A, \text { opt }}$ in the interval $\left[u_{n-1}, u_{n}[\right.$ :

$$
\frac{Z_{u_{n}-}^{A, \circ p t}}{Z_{u_{n-1}}^{A, \circ p t}}=\frac{Z_{u_{n}-}^{0}}{Z_{u_{n-1}}^{0}}=\frac{Z_{u_{n}}^{0}}{Z_{u_{n-1}}^{0}}
$$

and, more generally, for $t \in\left[u_{n-1}, u_{n}[\right.$

$$
\frac{Z_{t}^{A, o p t}}{Z_{u_{n-1}}^{A, o p t}}=\frac{Z_{t}^{0}}{Z_{u_{n-1}}^{0}}
$$

The next more delicate point comes with the (possible) jumps of $Z^{A, \text { opt }}$ at $u_{n-1}$. Defining again, for $I_{n-1} \in \operatorname{atom}\left(\mathcal{H}_{u_{n-1}}\right)$,

$$
a_{n-1}^{I_{n-1}}=\frac{\mathbf{E}\left[\left(\frac{Z^{A, \text { opt }}}{Z_{u_{n-1}}^{A, o p t}}\right)^{2} \mathbb{I} I_{n-1} \mid \mathcal{G}_{u_{n-1}-}^{A}\right]}{\mathbf{E}\left[\mathbb{I} I_{n-1} \mid \mathcal{G}_{u_{n-1}-}^{A}\right]^{2}}
$$

we may proceed analogously as above to calculate $\left(\hat{f}_{n-1}^{I_{n-1}}\right)_{I_{n-1} \in \operatorname{atom}\left(\mathcal{H}_{u_{n-1}}\right)}$.

Note that in the definition above we used the quotient

$$
\begin{aligned}
& \frac{Z_{\infty}^{A, o p t}}{Z_{u_{n-1}}^{A, \circ p t}}=\frac{Z_{\infty}^{A, o p t}}{Z_{u_{n}}^{A, \circ p t}} \cdot \frac{Z_{u_{n}}^{A, o p t}}{Z_{u_{n}-}^{A, o p t}} \cdot \frac{Z_{u_{n}-}^{A, o p t}}{Z_{u_{n-1}}^{A, \circ p t}} \\
& =\frac{Z_{\infty}^{0}}{Z_{u_{n}}^{0}} \cdot \frac{Z_{u_{n}, \text { opt }}^{A, o p t}}{Z_{u_{n}-}^{A, o p t}} \cdot \frac{Z_{u_{n}}^{0}}{Z_{u_{n-1}}^{0}} \\
& =\frac{Z_{\infty}^{0}}{Z_{u_{n-1}}^{0}} \cdot \frac{Z_{u_{n}}^{A, o p t}}{Z_{u_{n}-}^{A, o p t}},
\end{aligned}
$$

for which we need to know the relative jump of $Z_{u_{n}}^{A \text { opt }}$ which we calculated in the previous inductive step. This is the reason, why we have to use backward induction. 
Continuing in an obvious inductive way, we finally arrive at the ratio $\frac{Z_{\alpha}^{A, o p t}}{Z_{0}^{A, o p t}}$ which equals $Z_{\infty}^{A, o p t}$. Hence we obtain an (at least in theory) explicit way to calculate the density of the measure $\mathbb{Q}^{A, \text { opt }}$. Noting that by lemma 4.6 all the functions $\hat{f}_{i}^{I_{i}}$ are strictly positive on the support of $\mathbf{E}\left[\mathbb{1} I_{i} \mid \mathcal{G}_{u_{i}-}^{A}\right]$ we see that $Z^{A, o p t}$ is equivalent to $\mathbb{P}$ and we have, in particular, proved the subsequent proposition:

4.7 Proposition. Under the above assumption, the variance-optimal measure $\mathbb{Q}^{A, \text { opt }}$ for $R_{u}$ with respect to the filtration $\left(\mathcal{G}_{u}^{A}\right)_{u \in \mathbb{R}_{+}}$exists for every $A \in \mathcal{A}$ and is equivalent to $\mathbb{P}$.

In addition $\mathbb{Q}^{A, \text { opt }}$ may be calculated explicitly by backward induction.

q.e.d.

Next we turn to the behaviour of the family $\left(\mathbb{Q}^{A, o p t}\right)_{A \in \mathcal{A}}$ as $A$ increases along the partial order defined on $\mathcal{A}$.

4.8 Theorem. Under the above assumptions the following assertions are equivalent.

(i) The variance-optimal local martingale measure $\mathbb{Q}^{\text {opt }}$ for the process $S$ relative to the filtration $\left(\mathcal{F}_{t}\right)_{t \in \mathbb{R}_{+}}$ exists and is a $\mathbb{P}$-absolutely continuous probability measure, i.e., in $\mathcal{M}(\mathbb{P})$.

(ii) The family $\left(\mathbb{Q}^{A, \text { opt }}\right)_{A \in \mathcal{A}}$ remains bounded in $L^{2}(\mathbb{P})$.

(iii) The family $\left(\mathbb{Q}^{A, o p t}\right)_{A \in \mathcal{A}}$ converges in $L^{2}(\mathbb{P})$ along the partial order on $\mathcal{A}$. In this case the limit equals $\mathbb{Q}^{\text {opt }}$.

(iv) The constant function 1 is not in the $L^{2}(\mathbb{P})$-closure of $K_{0} \cap L^{2}(\mathbb{P})$.

If, in addition, the intersection of the $L^{2}(\mathbb{P})$-closure of $K_{0} \cap L^{2}(\mathbb{P})$ with $L^{2}(\mathbb{P})_{+}$is reduced to $\{0\}$ the measure $\mathbb{Q}^{\text {opt }}$ is equivalent to $\mathbb{P}$.

Proof. (i) $\Leftrightarrow$ (iv): The equivalence of (i) and (iv) follows from lemma 2.1 and theorem 3.1 .

As regards (ii) and (iii) denote, for $A \in \mathcal{A}$, by $K_{0}^{A}$ (resp. $K^{A}$ ) the subspace of $L^{2}(\mathbb{P}$ ) spanned by the simple stochastic integrals on $\left(R_{u}\right)_{u \in \mathbb{R}_{+}}$with respect to the filtration $\left(\mathcal{G}_{u}^{A}\right)_{u \in \mathbb{R}_{+}}$(resp. by $K_{0}^{A}$ and the constants). We know by proposition 4.4 above that $\left(K_{0}^{A}\right)_{A \in \mathcal{A}}\left(\operatorname{resp} .\left(K^{A}\right)_{A \in \mathcal{A}}\right)$ form a dense subspace of $K_{0}$ (resp. $K$ ) with respect to the norm of $L^{2}(\mathbb{P})$.

(i) $\Rightarrow$ (iii): Simply note that $\mathbb{Q}^{A, \text { opt }}$ is by lemma 2.1 the orthogonal projection of $\mathbb{Q}^{\text {opt }}$ onto the $L^{2}(\mathbb{P})$ closure of $K^{A}$.

(iii) $\Rightarrow$ (ii): Obvious, noting that for $B \geq A$

$$
\left\|\frac{d \mathbb{Q}^{B, \mathrm{opt}}}{d \mathbb{P}}\right\|_{L^{2}(\mathbb{P})} \geq\left\|\frac{d \mathbb{Q}^{A, \mathrm{opt}}}{d \mathbb{P}}\right\|_{L^{2}(\mathbb{P})}
$$

(ii) $\Rightarrow$ (i): This is an easy Hilbert space argument. For the convenience of the reader we isolate it in the subsequent lemma 4.9 .

The final assertion of the theorem follows from a theorem of Stricker ([St 90] th. 2) and the main theorem 1.3 .

q.e.d.

4.9 Lemma. Let $\left(K_{i}\right)_{i \in I}$ be an upward directed family of subspaces of a Hilbert space $H$ and $\left(x_{i}\right)_{i \in I}$ be elements of $K_{i}$ such that $K_{i} \subseteq K_{j}$ implies that $x_{i}$ equals the orthogonal projection of $x_{j}$ onto $K_{i}$.

If $\left(x_{i}\right)_{i \in I}$ is bounded in $H$ then $\left(x_{i}\right)_{i \in I}$ converges with respect to the norm of $H$ to an element $x_{0} \in H$ such that the orthogonal projection of $x_{0}$ onto $K_{i}$ equals $x_{i}$.

q.e.d.

Let us pause for a moment and recapitulate what we have achieved (resp. not achieved) in our attempt to give a satisfactory solution to problem 4.1 . 
First of all, we have not yet discretized the continuous process $\left(S_{t}\right)_{t \in \mathbb{R}_{+}}$. All we have done is to timechange the process $S$ to obtain a process $R_{u}=S_{T_{u}}$ which is adapted to the "natural Brownian filtration" $\left(\mathcal{G}_{u}\right)_{u \in \mathbb{R}_{+}}$so that we have a unique martingale measure $\mathbb{Q}^{0}$. Then we defined the family of "finite extensions" $\left(\mathcal{G}_{u}^{A}\right)_{u \in \mathbb{R}_{+}}$and gave a method to calculate the variance-optimal measures $\mathbb{Q}^{A, \text { opt }}$. Finally the $L^{2}(\mathbb{P})$-boundedness of the family $\left(\mathbb{Q}^{A, \text { opt }}\right)_{A \in \mathcal{A}}$ guarantees its convergence to the $\mathbb{P}$-absolutely continuous non-negative local martingale measure $\mathbb{Q}^{\text {opt }}$.

If we know in addition that $\mathcal{M}^{e}(\mathbb{P}) \cap L^{2}(\mathbb{P}) \neq \emptyset$, which is guaranteed by Stricker's "no free lunch" type condition $\hat{K}_{0} \cap L^{2}(\mathbb{P})_{+}=\{0\}$, we may conclude that $\mathbb{Q}^{\text {opt }}$ is in fact equivalent to $\mathbb{P}$.

We now modify the above construction to obtain the finite discretizations of $S$. We apply the most obvious way of discretising a continuous one-dimensional process by looking at the instances when it had moved by $n^{-1}$. We do this at a sufficiently large number of instances, e.g., $n^{3}$, to make sure that we follow the process all the time $t \in \mathbb{R}_{+}$as $n$ kinds to infinity. For $n \in \mathbb{N}$, define inductively the stopping times $\left(T_{i}^{(n)}\right)_{i=0}^{n^{3}}$ by $T_{0}=0$ and

$$
T_{i}^{(n)}=\inf \left\{t>T_{i-1}^{(n)}|| S_{t}-S_{T_{i-1}^{(n)}} \mid \geq n^{-1}\right\}
$$

It follows from our assumption $\lim _{t \rightarrow \infty}\langle S\rangle_{t}=\infty$ a.s. as well as from the existence of the equivalent martingale measure $\mathbb{Q}^{0}$ on $\mathcal{G}$ that each $T_{i}^{(n)}$ is almost surely finite and it is easy to verify that

$$
\lim _{n \rightarrow \infty} T_{n^{3}}^{(n)}=+\infty \quad \text { a.s. }
$$

Define the process $S^{(n)}=\left(S_{t}^{(n)}\right)_{t \in \mathbb{R}_{+}}$by

$$
S_{t}^{(n)}=S_{T_{i}^{(n)}}^{(n)}
$$

where $0 \leq i \leq n^{3}$ is the biggest number such that $T_{i}^{(n)} \leq t$. Denote by $R^{(n)}=\left(R_{i}^{(n)}\right)_{i=0}^{n^{3}}$ the process $\left(S_{T_{i}^{(n)}}^{(n)}\right)_{i=0}^{n^{3}}$ and by $\left(\mathcal{G}_{i}^{(n)}\right)_{i=0}^{n^{3}}$ the filtration generated by $R^{(n)}$. Obviously $R^{(n)}$ is a binomial process (scaled with step-size $n^{-1}$ ) and $\mathcal{G}^{(n)}=\mathcal{G}_{n^{3}}^{(n)}$ consists of $2^{n^{3}}$ atoms each having strictly positive $\mathbb{P}$-measure (under the above assumption on $S$ ). There is a unique equivalent martingale measure $\mathbb{Q}^{(n)}$ on $\mathcal{G}^{(n)}$ for $R$ which assigns to each atom the mass $2^{-n^{3}}$.

Now we define the finite extensions $\mathcal{G}_{i}^{A_{n}}$ of the filtration $\mathcal{G}_{i}^{(n)}$. We let $\mathcal{A}_{n}$ denote the set of all $A_{n}=$ $\left(\mathcal{H}_{1}^{(n)}, \ldots, \mathcal{H}_{n^{3}}^{(n)}\right)$, where $\left(\mathcal{H}_{i}^{(n)}\right)_{i=1}^{n^{3}}$ is an increasing sequence of finite $\sigma$-algebras contained in $\left(\mathcal{F}_{T_{i}^{(n)}}\right)_{i=1}^{n^{3}}$.

For each $A_{n} \in \mathcal{A}_{n}$ one may similarly as (and somewhat easier than) above calculate the variance-optimal extensions $\mathbb{Q}^{A_{n}, \text { opt }}$ of $\mathbb{Q}^{(n)}$ to the $\sigma$-algebra $\mathcal{G}^{A_{n}}=\mathcal{G}_{n^{3}}^{A_{n}}$. We refer to [Schw 94a] for an extensive treatment of the variance-optimal measure in finite discrete time.

Finally it should be clear how to proceed analogously as above to obtain the subsequent theorem 4.10 .

4.10 Theorem. Under the above assumptions the following assertions are equivalent.

(i) The variance-optimal local martingale measure $\mathbb{Q}^{\text {opt }}$ for the process $S$ relative to the filtration $\left(\mathcal{F}_{t}\right)_{t \in \mathbb{R}_{+}}$ exists and is a $\mathbb{P}$-absolutely continuous probability measure, i.e., in $\mathcal{M}(\mathbb{P})$.

(ii) The family $\left(\left(\mathbb{Q}^{A_{n}, \text { opt }}\right)_{A_{n} \in \mathcal{A}_{n}}\right)_{n \in \mathbb{N}}$ remains bounded in $L^{2}(\mathbb{P})$.

(iii) The family $\left(\left(\mathbb{Q}^{A_{n}, \circ p t}\right)_{A_{n} \in \mathcal{A}_{n}}\right)_{n \in \mathbb{N}}$ converges in $L^{2}(\mathbb{P})$ as $n$ tends to infinity and $A_{n}$ increases in $\mathcal{A}_{n}$. In this case the limit equals $\mathbb{Q}^{\text {opt }}$.

(iv) The constant function 1 is not in the $L^{2}(\mathbb{P})$-closure of $K_{0} \cap L^{2}(\mathbb{P})$. 
If, in addition, the intersection of the $L^{2}(\mathbb{P})$-closure of $K_{0} \cap L^{2}(\mathbb{P})$ with $L^{2}(\mathbb{P})_{+}$is reduced to $\{0\}$ the measure $\mathbb{Q}^{\text {opt }}$ is equivalent to $\mathbb{P}$.

We believe, that 4.10 gives quite a satisfactory solution to problem 4.1 in the case of continuous $\mathbb{R}$-valued processes $S$. Note that without the continuity assumption on $S$ there seems to be no hope for a reasonable solution to 4.1. On the other hand, it should be possible to extend the above construction to the case of continuous $\mathbb{R}^{d}$-valued processes. We leave this question as an open problem.

\section{REFERENCES}

[AS 92] J. P. Ansel, C. Stricker, Lois de Martingale, Densités at Décomposition de Föllmer Schweizer, Annales de L'Institut Henri Poincaré 28 (1992), 375-392.

[AS 93] J. P. Ansel, C. Stricker, Unicité et Existence de la loi minimale, Séminaire de Probablité, XXVII, LNM 1557 (1993), 22-29.

[DM 80] C. Dellacherie, P.A. Meyer, Probabilités et potentiel, Hermann, Paris, 1980.

[DR 91] D. Duffie, H.R. Richardson, Mean-Variance Hedging in Continuous Time, Annals of Applied Probability 1 (1991), 1-15.

[DS 94] F. Delbaen, W. Schachermayer, A General Version of the Fundamental Theorem of Asset Pricing, Math. Annalen 300 (1994), $463-520$.

[DS 95] F. Delbaen, W. Schachermayer, A simple counterexample to several problems in mathematical finance, which arises generically in incomplete markets, submitted.

[FS 91] H. Föllmer, M. Schweizer (1990), Hedging of Contingent Claims under Incomplete Information, appeared in M. H. A. Davis and R. J. Elliott (eds.): Applied Stochastic Analysis, Stochastic Monographs, Gordon and Breach, London/New York 5 (1991), 389 - 414.

[HK 79] M. J. Harrison, D. M. Kreps (1979), Martingales and arbitrage in multiperiod securities markets, J. Econ. Theory 20, 381-408.

[HP 81] M. J. Harrison, S. R. Pliska (1981), Martingales and stochastic integrals in the theory of continuous trading, Stoch. Proc. Appl. 11, 215-260.

[Ja 79] J. Jacod, Calcul stochastique et problémes de martingales, LNM 714, Springer, 1979.

[Kre 81] D. M. Kreps (1981), Arbitrage and Equilibrium in Economics with infinitely many Commodities, J. of Math. Econ. 8, 15-35.

[KS 91] I. Karatzas, S. Shreve, Brownian Motion and Stochastic Calculus, Springer Graduate Texts in Mathematics (1991).

[M 85] S. Müller, Arbitrage Pricing of Contingent Claims, Lecture Note in Economics and Mathematical Systems, Springer 254 (1985).

[RY 91] D. Revuz and M. Yor, Continuous Martingales and Brownian Motion, Springer Verlag, Berlin Heidelberg New York., 1991.

[Sch 93] W. Schachermayer, A counter-example to several problems in the theory of asset pricing, Math. Finance $\mathbf{3}$ (1993), 217-230.

[Schä 94] M. Schäl, On quadratic cost criteria for option hedging, Math. of Operations Research 19 (1994), $121-131$.

[Schw 92a] M. Schweizer, Mean-Variance Hedging for General Claims, Annals of Applied Probability 2 (1992), $171-179$.

[Schw 92b] M. Schweizer, Martingale Densities for General Asset Prices, Journal of Mathematical Economics 21 (1992), 363-378.

[Schw 94] M. Schweizer, Approximation Pricing and the Variance-Optimal Martingale Measure, preprint.

[Schw 94a] M. Schweizer, Variance-Optimal Hedging in Discrete Time, Mathematics of Operations Research, to appear.

[St 90] C. Stricker, Arbitrage et lois de martingale, Ann. Inst. H. Poincaré 26 (1990), 451-460.

[Yor 78] M. Yor, Sous-espaces denses dans $L^{1}$ ou $H^{1}$ et répresentation des martingales, Séminaire de Probabilité 
XII, LNM 649 (1978), 265-309.

Vrije Universiteit Brussel, Pleinlaan 2, B-1050 Brussels Belgium

E-mail: fdelbaen@tena2.vub.ac.be

Universität Wien, Brünnerstrasse 72, A-1210 Wien Austria

E-mail: wschach@ stat1.bwl.univie.ac.at 